\title{
RATIONAL DESIGN, SYNTHESIS AND
}

\author{
APPLICATION OF A NEW RECEPTOR FOR
}

\author{
THE MOLECULAR RECOGNITION OF
}

\section{TRICARBOXYLATE SALTS IN AQUEOUS}

\section{MEDIA}

Antonio Frontera*a, ${ }^{*}$, Jeroni Morey ${ }^{* a},{ }^{a}$ Antònia Oliver $^{a}$, M. Neus Piña ${ }^{a}$, David

Quiñonero $^{a}$, Antoni Costa ${ }^{a}$, Pablo Ballester ${ }^{b}$, Pere M. Deyà ${ }^{a}$ and Eric V. Anslyn ${ }^{* c}$

${ }^{a}$ Department of Chemistry, Universitat de les Illes Balears, Crta. de Valldemossa km

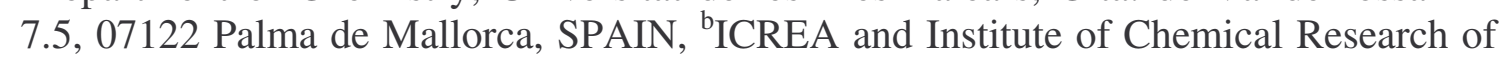
Catalonia (ICIQ), Avinguda Països Catalans 16, 43009 Tarragona, SPAIN and ${ }^{\mathrm{c}}$ Department of Chemistry and Biochemistry, University of Texas at Austin, 1 University Station, Austin, Texas 78712, USA.

\section{Supplementary material, Table of contents:}

Pages S2 to S40: Cartesian coordinates and energies of 1•G1-1•G5 complexes.

Pages S41 to S46: ITC binding curves of the data present in Table 1 of the manuscript, Figures S1-S6.

Pages S47-S49: ${ }^{1}$ H-NMR spectra of new species 1, 2 and 4 (Figures S7-S9). 
Cartesian coordinates of MARIJ-BP86/SVP optimized structure 1•G1 (endo, E=3340.243776 hartries):
C $\quad 0.8130 \quad 2.8251-1.9200$
C $1.9045 \quad 2.9124-1.0189$
C $\quad 1.6825 \quad 3.0296 \quad 0.3771$
$\begin{array}{llll}\text { C } & 0.3578 & 3.2004 & 0.8562\end{array}$
C $-0.7356 \quad 3.2389-0.0507$
C $-0.5062 \quad 3.0112-1.4322$
C $-1.6954 \quad 2.8961-2.3725$
C $0.0755 \quad 3.2337 \quad 2.3526$
C $3.3215 \quad 2.8125-1.5577$
$\mathrm{N}-2.4197 \quad 1.6318 \quad-2.1477$
$\begin{array}{llll}\mathrm{N} & -0.6108 & 1.9911 & 2.7432\end{array}$
$\mathrm{N} \quad 3.8153 \quad 1.4282-1.6168$
$\begin{array}{llll}\text { C }-2.1353 \quad 3.5838 & 0.4418\end{array}$
C $2.8664 \quad 2.9957 \quad 1.3345$
C $\quad 1.0625 \quad 2.5385-3.3964$
C $-3.6537 \quad 1.4118-2.6185$
C $-1.4836 \quad 1.8900 \quad 3.7524$
C $5.1106 \quad 1.1310-1.4793$
$\begin{array}{llll}\text { C } & -4.5650 & 0.3315 & -2.4648\end{array}$
C $-5.5164 \quad 0.9152-3.4366$
C $-4.6194 \quad 2.1725 \quad-3.4841$
C $-1.8773 \quad 2.7236 \quad 4.9413$
C $-2.7815 \quad 1.5376 \quad 5.3581$
C $-2.4410 \quad 0.8929 \quad 4.0713$
C $6.3279 \quad 1.8759-1.0085$
C $7.08730 .5359-1.1080$
C $5.8580-0.0650-1.6684$
$\begin{array}{llll}0 & -4.6216 & 3.2751 & -4.0126\end{array}$
$\begin{array}{lllll}0 & -6.5348 & 0.4807 & -3.9957\end{array}$
○ $6.5423 \quad 3.0203-0.6294$
$\begin{array}{lllll}0 & 8.1893 & 0.0702 & -0.7762\end{array}$
$\begin{array}{llll}0 & -1.5674 & 3.8268 & 5.3635\end{array}$
$\begin{array}{llll}0 & -3.4529 & 1.1811 & 6.3373\end{array}$ 

$\mathrm{N}-2.8010-0.2427 \quad 3.4547$
$\mathrm{N}-4.4962-0.8243-1.7886$
N $5.5035-1.2923-2.0772$
C $-5.5404-1.8212-1.8980$
C $6.4243-2.4065-2.0308$
C $-3.8042-1.1218 \quad 4.0133$
C $6.0691-3.4549-0.9706$
C $-3.2656-2.5012 \quad 4.3947$
C $-5.0763-3.1181-2.5636$
$\mathrm{N}-4.8997-3.0963-4.0920$
$\mathrm{N}-2.3776-2.5766 \quad 5.6473$
$\mathrm{N} \quad 6.4310 \quad-3.1303 \quad 0.4889$
$\begin{array}{llll}\mathrm{H} & -1.3741 & 2.9641 & -3.4298\end{array}$
$\mathrm{H}-2.4097 \quad 3.7306 \quad-2.2293$
$\mathrm{H}-0.5705 \quad 4.0874 \quad 2.6376$
H $1.0102 \quad 3.3560 \quad 2.9332$
$\mathrm{H} \quad 4.0395 \quad 3.3966-0.9520$
$\mathrm{H} \quad 3.3629 \quad 3.2566-2.5746$
$\begin{array}{llll}\mathrm{H} & -1.9277 & 0.8321 & -1.7016\end{array}$
H $-0.4883 \quad 1.1539 \quad 2.1445$
H $3.1522 \quad 0.6382-1.7850$
$\mathrm{H}-2.2876 \quad 3.2033 \quad 1.4681$
$\mathrm{H}-2.8932 \quad 3.0593-0.1697$
H $2.5916 \quad 2.4217 \quad 2.2438$
$\begin{array}{llll}\mathrm{H} & 3.6953 & 2.4221 & 0.8789\end{array}$
H $\quad 0.2582 \quad 1.8789 \quad-3.7796$
Н $\quad 1.9837 \quad 1.9356-3.5099$
H $-2.1892 \quad-0.5508 \quad 2.6287$
$\mathrm{H}-3.5130-1.0828-1.4039$
$\mathrm{H} \quad 4.4383-1.4548-2.1576$
H $-6.4260-1.3736-2.3934$
$\mathrm{H}-5.8741-2.1381-0.8821$
H $7.4606-2.0251-1.9269$
H $\quad 6.3882 \quad-2.9648 \quad-2.9944$
$\mathrm{H}-4.5937-1.3331 \quad 3.2553$ 

$\mathrm{H}-4.3075-0.6075 \quad 4.8572$
H $6.5940-4.4073-1.1876$
H $4.9626-3.5951-0.9616$
$\mathrm{H}-2.6175-2.8716 \quad 3.5665$
$\mathrm{H}-4.1056-3.1964 \quad 4.5949$
H $-5.8102-3.9270-2.3738$
$\mathrm{H}-4.0635-3.3835-2.1721$
$\mathrm{H} \quad 0.3470 \quad-3.4192-1.7899$
C $0.1003-2.3446-1.6721$
C $\quad 0.9611-1.7182-0.5316$
$\mathrm{H} \quad 0.4394-1.8345 \quad-2.6002$
C $-1.4311-2.2223-1.6463$
$\begin{array}{llll}0 & 0.7901 & -0.3109 & -0.4556\end{array}$
$\begin{array}{llll}\text { C } & 0.7396 & -2.3837 & 0.8533\end{array}$
C $2.4390-1.9796-0.9871$
$\begin{array}{llll}0 & -1.9514 & -1.1398 & -1.2027\end{array}$
$\begin{array}{llll}0 & -2.0891 & -3.1920 & -2.1371\end{array}$
$\mathrm{H} \quad 1.6342 \quad-2.1527 \quad 1.4714$
$\mathrm{H} \quad 0.7294 \quad-3.4855 \quad 0.7611$
C $-0.4568-1.9639 \quad 1.7216$
○ $3.1011-2.8986-0.4197$
○ $2.8668-1.2525-1.9478$
$\begin{array}{llll}0 & -0.9012 & -0.7593 & 1.6224\end{array}$
$\begin{array}{llll}0 & -0.8897 & -2.8165 & 2.5516\end{array}$
C $-3.1054-2.0694 \quad 6.8627$
$\mathrm{H}-2.4616 \quad-2.2287 \quad 7.7472$
$\mathrm{H}-3.3111-0.9823 \quad 6.7444$
$\mathrm{H}-4.0476-2.6366 \quad 6.9784$
C $-2.0127-4.0261 \quad 5.8126$
$\mathrm{H}-1.3712-4.1373 \quad 6.7057$
$\mathrm{H}-2.9345-4.6228 \quad 5.9343$
$\mathrm{H}-1.4675-4.3439 \quad 4.9051$
C $-1.0860-1.8051 \quad 5.4682$
$\mathrm{H}-0.6681-2.0956 \quad 4.4810$
$\mathrm{H}-1.3001-0.7250 \quad 5.5005$ 

H $-0.4166-2.0774 \quad 6.3049$
C $5.6460-1.94651 .0142$
H $6.0199-1.0266 \quad 0.5380$
H $\quad 4.5787-2.1178 \quad 0.7609$
$\mathrm{H} \quad 5.8126-1.8919 \quad 2.1059$
C $6.0265-4.3317 \quad 1.2972$
H $6.2655-4.1464 \quad 2.3603$
$\mathrm{H} \quad 4.9385-4.4784 \quad 1.1689$
H $\quad 6.5791-5.2180 \quad 0.9366$
$\begin{array}{llll}\text { C } \quad 7.9036 & -2.8597 & 0.6373\end{array}$
$\mathrm{H} \quad 8.1540-1.9110 \quad 0.1125$
H $8.1313-2.7532 \quad 1.7138$
$\begin{array}{llll}\mathrm{H} & 8.4696 & -3.7116 & 0.2168\end{array}$
C $-6.1760 \quad-2.7057-4.7854$
$\mathrm{H}-6.9856-3.3831-4.4555$
$\mathrm{H}-6.4203-1.6490-4.5386$
$\mathrm{H}-6.0264-2.8057-5.8763$
C $-4.4834-4.4886-4.4818$
$\mathrm{H}-4.3352-4.5248-5.5765$
$\mathrm{H}-3.5363-4.7155-3.9575$
$\mathrm{H}-5.2728-5.2024-4.1846$
C $-3.7853-2.1617-4.5132$
H $-4.0997-1.1199-4.3429$
$\mathrm{H}-2.8989-2.4174-3.8960$
$\mathrm{H}-3.6014-2.3243-5.5914$
C $3.3829 \quad 4.3890 \quad 1.7407$
H $2.6029 \quad 4.9786 \quad 2.2651$
$\mathrm{H} \quad 4.2619 \quad 4.3073 \quad 2.4114$
$\begin{array}{llll}\mathrm{H} & 3.6951 & 4.9751 & 0.8529\end{array}$
C $\quad 1.1618 \quad 3.7970 \quad-4.2798$
$\mathrm{H} \quad 1.3559 \quad 3.5276 \quad-5.3383$
Н $\quad 0.2274 \quad 4.3931-4.2469$
$\mathrm{H} \quad 1.9823 \quad 4.4643-3.9450$
C $-2.4085 \quad 5.10120 .4146$
$\mathrm{H}-2.3159 \quad 5.5141-0.6106$ 

$\mathrm{H}-3.4317 \quad 5.3253 \quad 0.7785$
$\mathrm{H}-1.6922 \quad 5.6543 \quad 1.0559$
$\mathrm{H}-0.0384-0.2052 \quad 0.0790$

Cartesian coordinates of the MARIJ-BP86/SVP optimized structure 1•G1 (exo, E=3340.240014 hartries):
C $\quad 0.6090 \quad 1.6854 \quad-3.2602$
C $1.6555 \quad 2.0903-2.3871$
C $\quad 1.3554 \quad 2.7197-1.1484$
C $\quad 0.0068 \quad 3.0388-0.8381$
C $-1.0311 \quad 2.7594-1.7665$
C $-0.7304 \quad 2.0538-2.9603$
C $-1.8789 \quad 1.5922 \quad-3.8514$
C $-0.3638 \quad 3.6037 \quad 0.5268$
C $3.1065 \quad 1.8518-2.7820$
$\begin{array}{llll}\mathrm{N} & -2.5639 & 0.4564 & -3.2228\end{array}$
$\begin{array}{llll}\mathrm{N} & -1.0970 & 2.5915 & 1.3043\end{array}$
$\mathrm{N} \quad 3.7344 \quad 0.6873 \quad-2.1339$
C $-2.4437 \quad 3.2680-1.5132$
C $2.4864 \quad 3.0945-0.1986$
C $\quad 0.9245 \quad 0.8794 \quad-4.5167$
$\begin{array}{llll}\text { C } & -3.8931 & 0.2760 & -3.2256\end{array}$
C $-1.9241 \quad 2.8867 \quad 2.3132$
C $5.0486 \quad 0.6543-1.8744$
C $-4.7190 \quad-0.6557-2.5513$
C $-5.9601-0.1496-3.1928$
C $-5.0790 \quad 0.9648-3.8332$
C $-2.3157 \quad 4.1276 \quad 3.0663$
C $-3.1341 \quad 3.1998 \quad 3.9939$
C $-2.8022 \quad 2.0798 \quad 3.0863$
C $6.1408 \quad 1.6838-1.8743$
C $7.0585 \quad 0.5917-1.2853$
C $5.9353-0.3640-1.4299$
$\begin{array}{llll}0 & -5.2285 & 1.9695 & -4.5154\end{array}$
$\begin{array}{llll}0 & -7.1352 & -0.5043 & -3.1822\end{array}$
○ $6.1953 \quad 2.8783-2.1382$ 

$\begin{array}{lllll}0 & 8.1891 & 0.5050 & -0.7847\end{array}$
$\begin{array}{llll}0 & -2.0408 & 5.3153 & 2.9754\end{array}$
$\begin{array}{llll}0 & -3.7452 \quad 3.2716 \quad 5.0713\end{array}$
$\begin{array}{llll}\mathrm{N} & -3.0924 & 0.7707 & 3.0476\end{array}$
$\mathrm{N}-4.3902-1.6401-1.6923$
N $5.7585-1.6571-1.1277$
C $-5.3117-2.5739-1.0954$
C $6.8194-2.4520-0.5453$
C $-3.9536 \quad 0.1553 \quad 4.0335$
C $6.5522-2.8626 \quad 0.9050$
C $-3.2209-0.8243 \quad 4.9549$
C $-4.5542-3.8109-0.6161$
$\mathrm{N}-4.1317-4.8321-1.6906$
$\begin{array}{llll}\mathrm{N} & -2.3747 & -0.2201 & 6.0888\end{array}$
N $6.7946-1.8038 \quad 1.9946$
H $-1.5084 \quad 1.3085-4.8568$
$\mathrm{H} \quad-2.6271 \quad 2.3894 \quad-4.0232$
$\begin{array}{llll}\mathrm{H} & -1.0023 & 4.5057 & 0.4447\end{array}$
$\mathrm{H} \quad 0.5384 \quad 3.9305 \quad 1.0802$
H $3.7376 \quad 2.7297 \quad-2.5417$
H $3.1782 \quad 1.7361-3.8827$
$\mathrm{H}-1.9917-0.2283-2.6866$
$\mathrm{H}-0.9554 \quad 1.5784 \quad 1.0958$
$\mathrm{H} \quad 3.1832-0.1649-1.9214$
$\mathrm{H}-2.6670 \quad 3.2575-0.4310$
$\mathrm{H}-3.1876 \quad 2.5858-1.9643$
H $2.1763 \quad 2.8949 \quad 0.8481$
H $3.3541 \quad 2.4310 \quad-0.3696$
H $\quad 0.1196 \quad 0.1362 \quad-4.6861$
$\begin{array}{llll}\mathrm{H} & 1.8337 & 0.2692 & -4.3482\end{array}$
$\begin{array}{llll}\mathrm{H} & -2.4688 & 0.1689 & 2.3984\end{array}$
$\mathrm{H}-3.3573-1.7343-1.4724$
$\mathrm{H} \quad 4.7250-1.9749-1.0503$
$\mathrm{H}-6.1576-2.7952-1.7851$
$\mathrm{H}-5.7969-2.1613-0.1752$ 

H $\quad 7.7875-1.9258-0.6722$
$\mathrm{H} \quad 6.9094-3.4172 \quad-1.0945$
$\mathrm{H}-4.7276-0.4652 \quad 3.5259$
$\mathrm{H}-4.5008 \quad 0.9439 \quad 4.5895$
$\mathrm{H} \quad 7.2068 \quad-3.7123 \quad 1.1863$
$\mathrm{H} \quad 5.4753-3.1294 \quad 1.0154$
H $-2.4938-1.4105 \quad 4.3415$
$\mathrm{H}-3.9497-1.4858 \quad 5.4663$
$\mathrm{H}-5.1593-4.3897 \quad 0.1084$
$\mathrm{H}-3.5947-3.5035-0.1360$
$\mathrm{H} \quad 1.3958-0.2083 \quad 1.4112$
C $\quad 1.0271-1.1947 \quad 1.7686$
C $\quad 1.2375-2.2372 \quad 0.6238$
$\mathrm{H} \quad 1.6621-1.5424 \quad 2.6041$
C $-0.4092-1.0085 \quad 2.2710$
$\begin{array}{llll}0 & 0.9520 & -3.5540 & 1.0748\end{array}$
C $0.4388-1.9022-0.6599$
C $\quad 2.7780 \quad-2.2271 \quad 0.3102$
$\begin{array}{llll}0 & -0.7393 & -1.5384 & 3.3729\end{array}$
$\begin{array}{llll}0 & -1.1918 & -0.2863 & 1.5456\end{array}$
H $\quad 0.9633-2.3956-1.5058$
$\mathrm{H} \quad 0.4676 \quad-0.8187-0.8839$
C $-1.0136-2.3612-0.7522$
○ $3.1464-1.9665-0.8906$
$\begin{array}{llll}0 & 3.5605 & -2.4879 & 1.2676\end{array}$
$\begin{array}{llll}0 & -1.4438 & -3.3083 & -0.0144\end{array}$
○ $-1.7243-1.8226-1.6725$
C $-3.2071 \quad 0.6621 \quad 6.9803$
$\mathrm{H}-2.5878 \quad 0.9686 \quad 7.8435$
$\mathrm{H}-3.5203 \quad 1.5654 \quad 6.4107$
$\begin{array}{llll}\mathrm{H} & -4.0853 & 0.0898 & 7.3329\end{array}$
C $-1.8209-1.3855 \quad 6.8616$
$\mathrm{H}-1.1914-1.0038 \quad 7.6861$
$\mathrm{H}-2.6562-1.9819 \quad 7.2711$
$\mathrm{H}-1.2148-1.9962 \quad 6.1671$ 

H $\quad 1.9376 \quad 2.4556-5.6750$
C $-2.6593 \quad 4.6883-2.0729$
$\mathrm{H}-2.4923 \quad 4.7213-3.1689$
$\mathrm{H}-3.6952 \quad 5.0324-1.8796$
$\mathrm{H}-1.9647 \quad 5.4191-1.6110$
$\begin{array}{llll}\mathrm{H} & -0.0211 & -3.6609 & 0.8847\end{array}$

Cartesian coordinates of the MARIJ-BP86/SVP optimized structure 1•G1 (exo,COSMO, $E=-3340.349612$ hartries):
C $0.7472 \quad 1.1218-3.4183$
C $\quad 1.7275 \quad 1.7325-2.5904$
C $1.3398 \quad 2.5693-1.5096$
C $-0.0326 \quad 2.8857-1.3335$
C $-1.0073 \quad 2.3861-2.2364$
C $-0.6187 \quad 1.4794-3.2559$
C $-1.6973 \quad 0.8162-4.1045$
C $-0.4940 \quad 3.6834-0.1221$
C $3.2042 \quad 1.4982-2.8710$
$\mathrm{N}-2.4080-0.2016-3.3177$
$\begin{array}{llll}\mathrm{N} & -1.1994 & 2.7965 & 0.8151\end{array}$
$\begin{array}{llll}\mathrm{N} & 3.8482 & 0.5021 & -1.9981\end{array}$
$\begin{array}{llll}\text { C } & -2.4439 & 2.8815 & -2.1557\end{array}$
C $2.4025 \quad 3.1602-0.5930$
$\begin{array}{llll}\text { C } & 1.1612 & 0.1152 & -4.4861\end{array}$
C $-3.7333-0.3583-3.3215$
C $-2.1695 \quad 3.1992 \quad 1.6363$
C $5.15750 .5548-1.7331$
C $-4.6041-1.1722-2.5305$
C $-5.8054-0.6839-3.2545$
$\begin{array}{llll}\text { C }-4.8854 & 0.2862 & -4.0032\end{array}$
C $-2.8509 \quad 4.48391 .9530$
C $-3.6513 \quad 3.7270 \quad 3.0138$
$\begin{array}{llll}\text { C }-3.0331 & 2.4751 & 2.5198\end{array}$
C $6.2372 \quad 1.5499-1.9790$
C $7.18620 .6177-1.2269$
C $6.0756-0.3592-1.1274$
$\begin{array}{llll}0 & -5.0131 & 1.2093 & -4.8157\end{array}$
$\begin{array}{llll}0 & -7.0146 & -0.9370 & -3.2174\end{array}$
$\begin{array}{llll}0 & 6.2961 & 2.6647 & -2.5072\end{array}$
$\begin{array}{llll}0 & 8.3628 & 0.6369 & -0.8350\end{array}$
$\begin{array}{llll}0 & -2.7633 & 5.6462 & 1.5430\end{array}$ 


$$
\begin{aligned}
& \begin{array}{llll}
0 & -4.4647 & 3.9989 & 3.9117
\end{array} \\
& \begin{array}{llll}
\mathrm{N} & -3.1261 & 1.1778 & 2.8011
\end{array} \\
& \mathrm{~N}-4.3269-2.0405-1.5570 \\
& \text { N } 5.9247-1.5754-0.6085 \\
& \text { C }-5.3065-2.7822-0.7788 \\
& \text { C } 7.0227-2.3315-0.0269 \\
& \begin{array}{llll}
\text { C }-4.0604 & 0.6376 & 3.7732
\end{array} \\
& \begin{array}{llll}
\text { C } & 6.8275 & -2.6891 & 1.4459
\end{array} \\
& \text { C }-3.3903-0.1192 \quad 4.9201 \\
& \begin{array}{llll}
\text { C }-4.6208 & -3.8503 & 0.0674
\end{array} \\
& \mathrm{~N}-4.2582-5.1630-0.6355 \\
& \begin{array}{llll}
\mathrm{N}-2.7305 & 0.7193 & 6.0188
\end{array} \\
& \text { N } 7.0680-1.5812 \quad 2.4752 \\
& \begin{array}{llll}
\mathrm{H} & -1.2582 & 0.3517 & -5.0071
\end{array} \\
& \mathrm{H}-2.4456 \quad 1.5426-4.4710 \\
& \mathrm{H}-1.1786 \quad 4.5075-0.3992 \\
& \begin{array}{llll}
\mathrm{H} & 0.3646 & 4.1621 & 0.3851
\end{array} \\
& \text { H } 3.7789 \quad 2.4374-2.7680 \\
& \mathrm{H} \quad 3.3377 \quad 1.1907-3.9264 \\
& \mathrm{H}-1.8625-0.7984-2.6556 \\
& \mathrm{H}-0.9387 \quad 1.7819 \quad 0.8655 \\
& \mathrm{H} \quad 3.3276-0.3298-1.6524 \\
& \mathrm{H}-2.7309 \quad 3.0581-1.1040 \\
& \mathrm{H}-3.1442 \quad 2.1075-2.5148 \\
& \text { H } 2.0398 \quad 3.1553 \quad 0.4538 \\
& \mathrm{H} \quad 3.2919 \quad 2.5055-0.5865 \\
& \mathrm{H} \quad 0.3974-0.6835-4.5544 \\
& \mathrm{H} \quad 2.0823-0.4094-4.1677 \\
& \begin{array}{llll}
\mathrm{H} & -2.3986 & 0.5459 & 2.3137
\end{array} \\
& \mathrm{H}-3.2950-2.1201-1.3041 \\
& \text { H } 4.9182-1.9354-0.5656 \\
& \mathrm{H}-6.1078-3.1913-1.4289 \\
& \mathrm{H}-5.8271-2.1162-0.0554 \\
& \text { H } 7.9729-1.7966-0.2139 \\
& \text { H } 7.1086-3.3095-0.5457 \\
& \mathrm{H}-4.7134-0.1157 \quad 3.2821 \\
& \mathrm{H}-4.7340 \quad 1.4422 \quad 4.1246 \\
& \mathrm{H} \quad 7.5265 \quad-3.4998 \quad 1.7194 \\
& \mathrm{H} \quad 5.7814 \quad-3.0169 \quad 1.6074 \\
& \mathrm{H}-2.5797-0.7574 \quad 4.5105 \\
& \mathrm{H}-4.1432-0.7411 \quad 5.4369
\end{aligned}
$$




$$
\begin{aligned}
& \begin{array}{llll}
\mathrm{H} & -5.2687 & -4.1419 & 0.9120
\end{array} \\
& \begin{array}{llll}
\mathrm{H} & -3.6613 & -3.4577 & 0.4657
\end{array} \\
& \begin{array}{llll}
\mathrm{H} & 1.5073 & 0.1096 & 1.2758
\end{array} \\
& \text { C } \quad 1.1533-0.7833 \quad 1.8319 \\
& \begin{array}{llll}
\text { C } & 1.3239 & -2.0380 & 0.9191
\end{array} \\
& \begin{array}{llll}
\mathrm{H} & 1.8142 & -0.9538 & 2.7019
\end{array} \\
& \begin{array}{llll}
\text { C } & -0.2741 & -0.5283 & 2.3336
\end{array} \\
& \begin{array}{llll}
0 & 0.9841 & -3.2267 & 1.6322
\end{array} \\
& \text { C } 0.5109-1.9460-0.3953 \\
& \begin{array}{llll}
\text { C } \quad 2.8622 & -2.1430 & 0.6224
\end{array} \\
& \begin{array}{llll}
0 & -0.5939 & -0.9370 & 3.4822
\end{array} \\
& \begin{array}{llll}
0 & -1.0623 & 0.1022 & 1.5329
\end{array} \\
& \text { H } \quad 1.0089-2.5890-1.1499 \\
& \text { H } \quad 0.5409-0.9229-0.8142 \\
& \text { C }-0.9540-2.3971-0.3625 \\
& \text { O } 3.2624-1.9401-0.5824 \\
& \begin{array}{llll}
0 & 3.6080 & -2.4267 & 1.5935
\end{array} \\
& \begin{array}{llll}
0 & -1.3830 & -3.1235 & 0.5877
\end{array} \\
& \begin{array}{llll}
0 & -1.6626 & -2.0810 & -1.3787
\end{array} \\
& \begin{array}{llll}
\text { C } & -3.7005 & 1.7012 & 6.6171
\end{array} \\
& \mathrm{H}-3.2033 \quad 2.2058 \quad 7.4624 \\
& \begin{array}{llll}
\mathrm{H} & -3.9880 & 2.4484 & 5.8557
\end{array} \\
& \mathrm{H}-4.5882 \quad 1.1530 \quad 6.9763 \\
& \begin{array}{llll}
\text { C } & -2.2750 & -0.2423 & 7.0858
\end{array} \\
& \begin{array}{llll}
\mathrm{H} & -1.7832 & 0.3287 & 7.8907
\end{array} \\
& \begin{array}{llll}
\mathrm{H} & -3.1523 & -0.7793 & 7.4819
\end{array} \\
& \begin{array}{llll}
\mathrm{H} & -1.5636 & -0.9541 & 6.6348
\end{array} \\
& \begin{array}{lll}
\text { C }-1.5162 & 1.4522 & 5.5013
\end{array} \\
& \begin{array}{llll}
\mathrm{H} & -0.8874 & 0.7261 & 4.9542
\end{array} \\
& \mathrm{H}-1.8402 \quad 2.2585 \quad 4.8262 \\
& \begin{array}{llll}
\mathrm{H} & -0.9843 & 1.8835 & 6.3659
\end{array} \\
& \begin{array}{llll}
\text { C } & 6.0572 & -0.4681 & 2.3380
\end{array} \\
& \begin{array}{llll}
\mathrm{H} & 6.2767 & 0.1063 & 1.4253
\end{array} \\
& \begin{array}{llll}
\mathrm{H} & 5.0521 & -0.9249 & 2.2742
\end{array} \\
& \begin{array}{llll}
\mathrm{H} & 6.1536 & 0.1897 & 3.2179
\end{array} \\
& \text { C } 6.8947-2.2062 \quad 3.8351 \\
& \text { H } \quad 7.0644-1.4339 \quad 4.6034 \\
& \text { H } \quad 5.8676 \quad-2.5998 \quad 3.9103 \\
& \text { H } \quad 7.6257 \quad-3.0234 \quad 3.9494 \\
& \text { C } 8.4591-1.0202 \quad 2.3579
\end{aligned}
$$




$$
\begin{array}{lrrr}
\text { H } & 8.5660 & -0.5035 & 1.3870 \\
\mathrm{H} & 8.6117 & -0.2998 & 3.1787 \\
\mathrm{H} & 9.1853 & -1.8466 & 2.4423 \\
\mathrm{C} & -5.4946 & -5.9768 & -0.8944 \\
\mathrm{H} & -5.9803 & -6.2037 & 0.0691 \\
\mathrm{H} & -6.1792 & -5.4011 & -1.5386 \\
\mathrm{H} & -5.2021 & -6.9109 & -1.4020 \\
\mathrm{C} & -3.3424 & -5.9181 & 0.2954 \\
\mathrm{H} & -3.0928 & -6.8882 & -0.1654 \\
\mathrm{H} & -2.4376 & -5.3032 & 0.4450 \\
\mathrm{H} & -3.8644 & -6.0759 & 1.2540 \\
\mathrm{C} & -3.5315 & -4.9311 & -1.9356 \\
\mathrm{H} & -4.2283 & -4.4886 & -2.6648 \\
\mathrm{H} & -2.6845 & -4.2456 & -1.7588 \\
\mathrm{H} & -3.1765 & -5.9078 & -2.3055 \\
\mathrm{C} & 2.8248 & 4.5904 & -0.9770 \\
\mathrm{H} & 1.9711 & 5.2949 & -0.9275 \\
\mathrm{H} & 3.6143 & 4.9647 & -0.2961 \\
\mathrm{H} & 3.2227 & 4.6300 & -2.0101 \\
\mathrm{C} & 1.3828 & 0.7251 & -5.8820 \\
\mathrm{H} & 1.6807 & -0.0538 & -6.6107 \\
\mathrm{H} & 0.4643 & 1.2132 & -6.2626 \\
\mathrm{H} & 2.1781 & 1.4968 & -5.8657 \\
\mathrm{C} & -2.6602 & 4.1701 & -2.9731 \\
\mathrm{H} & -2.4302 & 4.0100 & -4.0449 \\
\mathrm{H} & -3.7113 & 4.5118 & -2.8987 \\
\mathrm{H} & -2.0087 & 4.9925 & -2.6168 \\
\mathrm{H} & -0.0024 & -3.3168 & 1.4544
\end{array}
$$

Cartesian coordinates of the MARIJ-BP86/SVP optimized structure 1•G2 (endo, E=3265.064962 hartries):
C $\quad 1.3764-0.6890-3.5310$
C $2.4058 \quad 0.0980-2.9448$
C $2.1066 \quad 1.3659-2.3771$
C $\quad 0.7901 \quad 1.8833-2.4921$
C $-0.2067 \quad 1.1709-3.2117$
C $0.0786-0.1327-3.6949$
C $-1.0591-0.9756-4.2672$
C $0.3841 \quad 3.1537-1.7591$
C $3.8328-0.4335-2.8912$ 

$\mathrm{N}-1.8971-1.4936-3.1776$
$\mathrm{N}-0.5437 \quad 2.8000 \quad-0.6674$
N $4.2129-0.9664-1.5728$
C $-1.5413 \quad 1.8327-3.5258$
C $3.2066 \quad 2.1847-1.7135$
C $\quad 1.6659-2.1143-3.9932$
C $-3.2252-1.3157-3.1094$
C $-1.3718 \quad 3.6649-0.0659$
C $5.4130-0.7383-1.0283$
C $-4.1633-1.5203-2.0660$
C $-5.3070-1.0549-2.8908$
C $-4.2737-0.7256-4.0024$
C $-1.4885 \quad 5.16330 .0086$
C $-2.6066 \quad 4.9296 \quad 1.0558$
C $-2.5037 \quad 3.4804 \quad 0.7661$
C $6.6042 \quad 0.0981-1.4010$
C $7.1916-0.2686-0.0226$
C $6.0198-1.14690 .1916$
$0-4.2518-0.1916-5.1065$
$\begin{array}{lllll}0 & -6.5181 & -0.9664 & -2.6971\end{array}$
$\begin{array}{llll}0 & 6.8827 & 0.8221 & -2.3484\end{array}$
$\begin{array}{llll}0 & 8.1407 & 0.0758 & 0.6977\end{array}$
$0-0.8967 \quad 6.0997-0.5039$
$\begin{array}{llll}0 & -3.2585 & 5.5837 & 1.8808\end{array}$
$\begin{array}{llll}\mathrm{N} & -3.1527 & 2.3933 & 1.2149\end{array}$
$\mathrm{N}-4.0097-1.9920-0.8149$
N $5.5827-1.9136 \quad 1.2016$
C $-5.0146-1.81650 .2088$
C $6.3157-2.0233 \quad 2.4437$
$\begin{array}{lll}\text { C }-4.2766 \quad 2.5392 & 2.1215\end{array}$
C $5.6207-1.3442 \quad 3.6290$
C $-4.0586 \quad 1.8112 \quad 3.4425$
C $-4.7562-2.7245 \quad 1.4060$
$\mathrm{N}-5.1383-4.2080 \quad 1.2532$
$\mathrm{N}-3.0521 \quad 2.4380 \quad 4.4222$ 


$$
\begin{aligned}
& \begin{array}{llll}
\mathrm{N} & 5.6996 & 0.1891 & 3.7104
\end{array} \\
& \mathrm{H}-0.6606-1.8160-4.8698 \\
& \mathrm{H}-1.7141-0.3967-4.9450 \\
& \mathrm{H}-0.1260 \quad 3.8726-2.4314 \\
& \mathrm{H} \quad 1.2623 \quad 3.6913-1.3541 \\
& \text { H } 4.5686 \quad 0.3549-3.1445 \\
& \text { H } 3.9706-1.2238-3.6560 \\
& \text { H }-1.4308-1.8663-2.3264 \\
& \mathrm{H}-0.6077 \quad 1.8084-0.3940 \\
& \text { Н } \quad 3.5169-1.4734-0.9888 \\
& \mathrm{H}-1.8625 \quad 2.4786 \quad-2.6881 \\
& \mathrm{H}-2.3416 \quad 1.0808-3.6337 \\
& \mathrm{H} \quad 2.8099 \quad 2.6739-0.8001 \\
& \text { H } 4.0121 \quad 1.5190-1.3549 \\
& \mathrm{H} \quad 0.7791-2.7509-3.8002 \\
& \text { H } \quad 2.4635 \quad-2.5580 \quad-3.3647 \\
& \mathrm{H}-2.6254 \quad 1.4596 \quad 1.1865 \\
& \mathrm{H}-2.9954-2.2289-0.5485 \\
& \mathrm{H} \quad 4.5277 \quad-2.1528 \quad 1.1619 \\
& \mathrm{H}-6.0321-1.9156-0.2326 \\
& \begin{array}{llll}
\mathrm{H} & -4.9851 & -0.7839 & 0.6387
\end{array} \\
& \text { H } \quad 7.3542-1.6638 \quad 2.2937 \\
& \text { H } \quad 6.3922 \quad-3.0934 \quad 2.7452 \\
& \mathrm{H}-5.1910 \quad 2.0693 \quad 1.6882 \\
& \mathrm{H}-4.5080 \quad 3.6184 \quad 2.2423 \\
& \mathrm{H} \quad 6.0623-1.7038 \quad 4.5804 \\
& \text { H } \quad 4.5292-1.5627 \quad 3.5898 \\
& \begin{array}{llll}
\mathrm{H} & -3.6513 & 0.7837 & 3.2418
\end{array} \\
& \mathrm{H}-5.0078 \quad 1.7474 \quad 4.0116 \\
& \mathrm{H}-5.3230 \quad-2.3714 \quad 2.2888 \\
& \mathrm{H}-3.6748 \quad-2.7234 \quad 1.6537 \\
& \text { H } \quad 0.5411-3.9251 \quad 1.6986 \\
& \text { C } \quad 0.3542-3.1740 \quad 0.9041 \\
& \text { C } 0.6992-1.7561 \quad 1.4206 \\
& \begin{array}{llll}
\mathrm{H} & 1.0205 & -3.3986 & 0.0482
\end{array}
\end{aligned}
$$



C $-1.1028-3.2762 \quad 0.4537$
H $0.5046-1.0446 \quad 0.5863$
C $-0.1727-1.3254 \quad 2.6120$
C $2.2209-1.6596 \quad 1.7006$
$\begin{array}{llll}0 & -1.4359 & -2.5902 & -0.5732\end{array}$
$\begin{array}{llll}0 & -1.9075 & -3.9867 & 1.1311\end{array}$
H $\quad 0.4644-0.6881 \quad 3.2649$
H $-0.4765-2.1955 \quad 3.2280$
C $-1.4351-0.5014 \quad 2.3067$
$\begin{array}{llll}0 & 2.6298 & -1.1284 & 2.7778\end{array}$
$\begin{array}{llll}0 & 2.9879 & -2.1382 & 0.7895\end{array}$
$\begin{array}{llll}0 & -1.3765 & 0.3780 & 1.3772\end{array}$
$\begin{array}{llll}0 & -2.4432 & -0.6799 & 3.0663\end{array}$
C $-3.5754 \quad 3.7381 \quad 4.9655$
$\mathrm{H}-2.8569 \quad 4.1269 \quad 5.7108$
$\mathrm{H}-3.6686 \quad 4.4615 \quad 4.1300$
$\mathrm{H}-4.5556 \quad 3.5624 \quad 5.4452$
C $-2.8661 \quad 1.4204 \quad 5.5185$
$\mathrm{H}-2.1428 \quad 1.8130 \quad 6.2568$
$\mathrm{H}-3.8413 \quad 1.2397 \quad 6.0061$
H $-2.5000 \quad 0.4885 \quad 5.0397$
C $-1.7018 \quad 2.6982 \quad 3.7865$
$\mathrm{H}-1.4115 \quad 1.8397 \quad 3.1530$
$\mathrm{H}-1.7703 \quad 3.6085 \quad 3.1682$
$\mathrm{H}-0.9749 \quad 2.8601 \quad 4.6035$
$\begin{array}{llll}\text { C } & 4.9678 & 0.8501 & 2.5602\end{array}$
H $5.5506 \quad 0.7133 \quad 1.6356$
$\begin{array}{llll}\mathrm{H} & 3.9716 & 0.3626 & 2.4812\end{array}$
$\mathrm{H} \quad 4.8890 \quad 1.9286 \quad 2.7900$
C $4.9872 \quad 0.5780 \quad 4.9762$
$\mathrm{H} \quad 5.0150 \quad 1.6780 \quad 5.0795$
H $3.9425 \quad 0.2254 \quad 4.8999$
$\begin{array}{llll}\mathrm{H} & 5.4908 & 0.1068 & 5.8397\end{array}$
$\begin{array}{llll}\text { C } \quad 7.1280 & 0.6614 & 3.7403\end{array}$
$\mathrm{H} \quad 7.6095 \quad 0.4370 \quad 2.7626$ 

$\mathrm{H} \quad 7.1295 \quad 1.7543 \quad 3.9071$
$\mathrm{H} \quad 7.6560 \quad 0.1546 \quad 4.5692$
C $-6.6279-4.3600 \quad 1.2693$
$\mathrm{H}-7.0238-3.9775 \quad 2.2280$
$\mathrm{H}-7.0607-3.7869 \quad 0.4300$
$\mathrm{H} \quad-6.8831-5.4294 \quad 1.1574$
C $-4.5204-4.9330 \quad 2.4238$
$\mathrm{H}-4.8171-5.9968 \quad 2.3777$
$\mathrm{H}-3.4214-4.8165 \quad 2.3303$
$\mathrm{H}-4.8897-4.4758 \quad 3.3600$
C $-4.5743-4.8157-0.0133$
$\mathrm{H}-5.0146-4.3094-0.8873$
$\mathrm{H}-3.4745-4.6545 \quad 0.0336$
$\mathrm{H}-4.8345-5.8912-0.0085$
C $\quad 3.8177 \quad 3.2503-2.6433$
Н $\quad 3.0579 \quad 3.9861-2.9765$
$\mathrm{H} \quad 4.6323 \quad 3.8022-2.1328$
$\mathrm{H} \quad 4.2512 \quad 2.7862-3.5516$
C $\quad 2.0641-2.2329-5.4768$
$\mathrm{H} \quad 2.2613-3.2887-5.7550$
$\mathrm{H} \quad 1.2657-1.8488-6.1443$
$\mathrm{H} \quad 2.9788-1.6460-5.6995$
C $-1.4805 \quad 2.6617-4.8246$
$\mathrm{H}-1.2085 \quad 2.0272-5.6921$
$\mathrm{H}-2.4661 \quad 3.1199-5.0407$
$\mathrm{H}-0.7279 \quad 3.4748-4.7602$

Cartesian coordinates of the MARIJ-BP86/SVP optimized structure 1•G2 (exo, E=3265.067578 hartries):
C $\quad 1.2179-0.7106-3.4515$
C $2.2083 \quad 0.0737-2.7989$
C $\quad 1.8705 \quad 1.3329-2.2330$
C $\quad 0.5579 \quad 1.8457-2.4088$
C $-0.4028 \quad 1.1233-3.1660$
C $-0.0772-0.1653-3.6636$
C $-1.1666-0.9996-4.3315$ 

C $0.1312 \quad 3.1412-1.7317$
C $3.6425-0.4333-2.7170$
$\mathrm{N}-2.0594-1.5752-3.3193$
$\mathrm{N}-0.8201 \quad 2.8509-0.6454$
$\mathrm{N} \quad 4.0252 \quad-0.9998-1.4117$
C $-1.7459 \quad 1.7562-3.5021$
C $2.9328 \quad 2.1425-1.4992$
C $1.5478-2.1203-3.9350$
C $-3.3981-1.5278-3.3902$
C $-1.6078 \quad 3.7696-0.0754$
C $5.2616-0.8525-0.9200$
C $-4.4034-1.8725 \quad-2.4544$
C $-5.5106-1.5067-3.3770$
C $-4.4130-1.0220-4.3698$
C $-1.7455 \quad 5.2676-0.1234$
C $-2.7980 \quad 5.1180 \quad 1.0010$
C $-2.6754 \quad 3.6525 \quad 0.8554$
C $6.4483-0.0151-1.3021$
C $7.1212-0.5217-0.0111$
C $5.9380-1.3842 \quad 0.2130$
$\begin{array}{llll}0 & -4.3450 & -0.4505 & -5.4513\end{array}$
$\begin{array}{llll}0 & -6.7347 & -1.5628 & -3.3142\end{array}$
$\begin{array}{llll}0 & 6.6840 & 0.7812 & -2.2023\end{array}$
$\begin{array}{llll}0 & 8.1353 & -0.2759 & 0.6592\end{array}$
$\begin{array}{llll}0 & -1.1994 & 6.1653 & -0.7466\end{array}$
$\begin{array}{llll}0 & -3.4285 & 5.8460 & 1.7842\end{array}$
$\mathrm{N}-3.2452 \quad 2.5915 \quad 1.4473$
$\mathrm{N}-4.2792-2.3686-1.2080$
N $5.5653-2.2362 \quad 1.1786$
C $-5.3586-2.5386-0.2664$
$\begin{array}{llll}\text { C } & 6.4049 & -2.4888 & 2.3308\end{array}$
C $-4.2705 \quad 2.7436 \quad 2.4560$
C $5.8437-1.9170 \quad 3.6376$
$\begin{array}{lll}\text { C }-3.8181 & 2.3119 & 3.8540\end{array}$
C $-4.8344-3.2020 \quad 1.0045$ 


$$
\begin{aligned}
& \mathrm{N}-4.5651-4.7189 \quad 0.9447 \\
& \mathrm{~N}-2.9604 \quad 3.3080 \quad 4.6536 \\
& \text { N } 6.0094 \quad-0.4068 \quad 3.8804 \\
& \mathrm{H}-0.7190-1.8066-4.9465 \\
& \mathrm{H}-1.7879-0.4012-5.0241 \\
& \text { H }-0.3559 \quad 3.8324-2.4488 \\
& \text { H } \quad 1.0045 \quad 3.6938-1.3347 \\
& \text { H } \quad 4.3657 \quad 0.3774 \quad-2.9339 \\
& \text { H } 3.8142-1.1957-3.5033 \\
& \text { H }-1.6448-1.9520-2.4386 \\
& \mathrm{H}-0.8819 \quad 1.8823-0.2602 \\
& \text { H } 3.3514-1.5561-0.8511 \\
& \mathrm{H}-2.0881 \quad 2.3948 \quad-2.6669 \\
& \mathrm{H}-2.5259 \quad 0.9817-3.6107 \\
& \text { H } 2.4809 \quad 2.6321-0.6122 \\
& \text { H } 3.7035 \quad 1.4644-1.0889 \\
& \text { H } \quad 0.6604-2.7713-3.8021 \\
& \mathrm{H} \quad 2.3186-2.5698-3.2775 \\
& \mathrm{H}-2.7213 \quad 1.6558 \quad 1.3344 \\
& \mathrm{H}-3.2766-2.4685-0.8590 \\
& \mathrm{H} \quad 4.5095 \quad-2.4752 \quad 1.2053 \\
& \text { H }-6.2239-3.0715-0.7249 \\
& \mathrm{H}-5.7801-1.5599 \quad 0.0729 \\
& \mathrm{H} \quad 7.4377-2.1446 \quad 2.1178 \\
& \mathrm{H} \quad 6.4691 \quad-3.5859 \quad 2.5152 \\
& \mathrm{H} \quad-5.1324 \quad 2.0767 \quad 2.2221 \\
& \text { H }-4.6625 \quad 3.7805 \quad 2.4291 \\
& \mathrm{H} \quad 6.3386-2.3989 \quad 4.5049 \\
& \mathrm{H} \quad 4.7404 \quad-2.0812 \quad 3.6582 \\
& \begin{array}{llll}
\mathrm{H} & -3.1741 & 1.4032 & 3.7564
\end{array} \\
& \mathrm{H}-4.6988 \quad 2.1186 \quad 4.4993 \\
& \mathrm{H} \quad-5.5491 \quad-3.0719 \quad 1.8397 \\
& \mathrm{H}-3.8470-2.7628 \quad 1.2850 \\
& \text { H } \quad 1.0917 \quad 0.5757 \quad 1.3565 \\
& \begin{array}{llll}
\text { C } & 0.5840 & -0.0392 & 2.1328
\end{array}
\end{aligned}
$$



C $0.8129-1.5358 \quad 1.7963$
H $1.0630 \quad 0.1554 \quad 3.1106$
C $-0.8806 \quad 0.4156 \quad 2.2085$
H $\quad 0.2956-2.1309 \quad 2.5822$
C $0.2499-1.9770 \quad 0.4419$
C $2.3269-1.8403 \quad 1.9578$
$\begin{array}{llll}0 & -1.3988 & 0.5735 & 3.3556\end{array}$
$\begin{array}{llll}0 & -1.4810 & 0.6507 & 1.0970\end{array}$
H $\quad 0.9009 \quad-2.7815 \quad 0.0379$
H $\quad 0.2849-1.1608-0.3093$
$\begin{array}{llll}\text { C } & -1.1706 & -2.5423 & 0.4455\end{array}$
$\begin{array}{llll}0 & 2.9383 & -2.4012 & 0.9794\end{array}$
$\begin{array}{llll}0 & 2.8740 & -1.5140 \quad 3.0552\end{array}$
$\begin{array}{llll}0 & -1.7464 & -2.8362 & 1.5292\end{array}$
$\begin{array}{llll}0 & -1.6903 & -2.7476 & -0.7199\end{array}$
C $-3.6818 \quad 4.6134 \quad 4.8479$
$\mathrm{H}-3.0869 \quad 5.2425 \quad 5.5354$
$\mathrm{H}-3.7694 \quad 5.1240 \quad 3.8642$
$\mathrm{H}-4.6768 \quad 4.4145 \quad 5.2882$
C $-2.6779 \quad 2.6452 \quad 5.9733$
H $-2.0587 \quad 3.3215 \quad 6.5906$
$\mathrm{H}-3.6325 \quad 2.4348 \quad 6.4887$
$\mathrm{H}-2.1337 \quad 1.7046 \quad 5.7669$

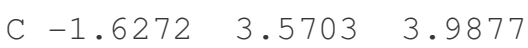
$\mathrm{H}-1.1722 \quad 2.5826 \quad 3.7675$
$\mathrm{H}-1.7929 \quad 4.1507 \quad 3.0665$
$\mathrm{H}-1.0135 \quad 4.1624 \quad 4.6918$
C $5.2436 \quad 0.4149 \quad 2.8648$
$\mathrm{H} \quad 5.7529 \quad 0.3480 \quad 1.8906$
$\mathrm{H} \quad 4.2167-0.0046 \quad 2.8111$
$\mathrm{H} \quad 5.2483 \quad 1.4646 \quad 3.2115$
$\begin{array}{llll}\text { C } & 5.4002 & -0.1321 & 5.2274\end{array}$
$\mathrm{H} \quad 5.4932 \quad 0.9466 \quad 5.4491$
$\mathrm{H} \quad 4.3350 \quad-0.4249 \quad 5.1832$
H $5.9312-0.7220 \quad 5.9963$ 


$$
\begin{aligned}
& \text { C } 7.4592-0.0065 \quad 3.8583 \\
& \mathrm{H} \quad 7.8556 \quad-0.1359 \quad 2.8272 \\
& \mathrm{H} \quad 7.5317 \quad 1.0584 \quad 4.1469 \\
& \mathrm{H} \quad 8.0163 \quad-0.6296 \quad 4.5824 \\
& \begin{array}{llll}
\text { C }-5.8583 & -5.4766 & 0.9485
\end{array} \\
& \mathrm{H}-6.4085-5.2536 \quad 1.8807 \\
& \text { H }-6.4611-5.1699 \quad 0.0751 \\
& \mathrm{H}-5.6428-6.5588 \quad 0.8901 \\
& \text { C }-3.7534 \quad-5.0543 \quad 2.1741 \\
& \mathrm{H}-3.5473-6.1402 \quad 2.1762 \\
& \mathrm{H}-2.8213-4.4475 \quad 2.1156 \\
& \mathrm{H}-4.3401-4.7781 \quad 3.0695 \\
& \text { C }-3.7567-5.1124-0.2729 \\
& \mathrm{H}-4.3808-4.9840-1.1729 \\
& \mathrm{H}-2.8656-4.4507-0.3488 \\
& \mathrm{H}-3.4685-6.1734-0.1521 \\
& \text { C } \quad 3.6168 \quad 3.2059-2.3797 \\
& \text { H } \quad 2.8867 \quad 3.9444 \quad-2.7689 \\
& \mathrm{H} \quad 4.3904 \quad 3.7561-1.8070 \\
& \mathrm{H} \quad 4.1186 \quad 2.7430 \quad-3.2524 \\
& \text { C } 2.0215-2.1982-5.3990 \\
& \mathrm{H} \quad 2.2397-3.2454-5.6936 \\
& \text { Н } \quad 1.2551-1.8019-6.0958 \\
& \text { H } \quad 2.9421-1.6010-5.5605 \\
& \text { C }-1.6910 \quad 2.5850-4.8010 \\
& \mathrm{H}-1.3957 \quad 1.9582 \quad-5.6665 \\
& \mathrm{H}-2.6836 \quad 3.0224 \quad-5.0279 \\
& \mathrm{H}-0.9579 \quad 3.4147-4.7283
\end{aligned}
$$

Cartesian coordinates of the MARIJ-BP86/SVP optimized structure 1•G2 (exo, COSMO, $E=-3265.173088$ hartries):
C $1.2179-0.7106-3.4515$
C $2.20830 .0737-2.7989$
C $1.8705 \quad 1.3329-2.2330$
C $0.5579 \quad 1.8457-2.4088$
C $-0.40281 .1233-3.1660$
C $-0.0772-0.1653-3.6636$ 

C $-1.1666-0.9996-4.3315$
C $0.1312 \quad 3.1412-1.7317$
C $3.6425-0.4333-2.7170$
$\mathrm{N}-2.0594-1.5752-3.3193$
$\mathrm{N}-0.8201 \quad 2.8509-0.6454$
$\mathrm{N} \quad 4.0252-0.9998-1.4117$
C $-1.7459 \quad 1.7562-3.5021$
C $2.93282 .1425-1.4992$
C $1.5478-2.1203-3.9350$
C $-3.3981-1.5278-3.3902$
C $-1.6078 \quad 3.7696-0.0754$
C $5.2616-0.8525-0.9200$
C $-4.4034-1.8725-2.4544$
C $-5.5106-1.5067-3.3770$
C $-4.4130-1.0220-4.3698$
C $-1.7455 \quad 5.2676-0.1234$
C $-2.7980 \quad 5.1180 \quad 1.0010$
C $-2.6754 \quad 3.6525 \quad 0.8554$
C $6.4483-0.0151-1.3021$
C $7.1212-0.5217-0.0111$
C $5.9380-1.38420 .2130$
$0-4.3450-0.4505-5.4513$
○ $-6.7347-1.5628-3.3142$
$\begin{array}{llll}0 & 6.6840 & 0.7812 & -2.2023\end{array}$
$\begin{array}{llll}0 & 8.1353 & -0.2759 & 0.6592\end{array}$
$0-1.19946 .1653-0.7466$
$\begin{array}{llll}0 & -3.4285 & 5.8460 & 1.7842\end{array}$
$\mathrm{N}-3.2452 \quad 2.5915 \quad 1.4473$
$\mathrm{N}-4.2792-2.3686-1.2080$
$\begin{array}{llll}\mathrm{N} & 5.5653 & -2.2362 & 1.1786\end{array}$
C $-5.3586-2.5386-0.2664$
$\begin{array}{llll}\text { C } & 6.4049 & -2.4888 & 2.3308\end{array}$
C $-4.2705 \quad 2.7436 \quad 2.4560$
C $5.8437-1.9170 \quad 3.6376$
$\begin{array}{lll}\text { C }-3.8181 & 2.3119 & 3.8540\end{array}$
C $-4.8344-3.2020 \quad 1.0045$
$\begin{array}{llll}\mathrm{N} & -4.5651 & -4.7189 & 0.9447\end{array}$
$\mathrm{N}-2.9604 \quad 3.3080 \quad 4.6536$
$\mathrm{N} \quad 6.0094-0.4068 \quad 3.8804$
$\mathrm{H}-0.7190-1.8066-4.9465$
$\mathrm{H}-1.7879-0.4012-5.0241$ 


$$
\begin{aligned}
& \mathrm{H}-0.3559 \quad 3.8324-2.4488 \\
& \mathrm{H} \quad 1.0045 \quad 3.6938-1.3347 \\
& \text { H } 4.3657 \quad 0.3774 \quad-2.9339 \\
& \mathrm{H} \quad 3.8142-1.1957-3.5033 \\
& \mathrm{H}-1.6448-1.9520-2.4386 \\
& \mathrm{H}-0.8819 \quad 1.8823-0.2602 \\
& \text { Н } 3.3514-1.5561-0.8511 \\
& \mathrm{H}-2.08812 .3948-2.6669 \\
& \mathrm{H}-2.5259 \quad 0.9817-3.6107 \\
& \text { H } 2.4809 \quad 2.6321-0.6122 \\
& \mathrm{H} \quad 3.7035 \quad 1.4644-1.0889 \\
& \mathrm{H} \quad 0.6604-2.7713-3.8021 \\
& \text { H } 2.3186-2.5698-3.2775 \\
& \mathrm{H}-2.7213 \quad 1.6558 \quad 1.3344 \\
& \mathrm{H}-3.2766-2.4685-0.8590 \\
& \text { H } 4.5095-2.4752 \quad 1.2053 \\
& \text { H }-6.2239-3.0715-0.7249 \\
& \mathrm{H}-5.7801-1.5599 \quad 0.0729 \\
& \text { H } 7.4377-2.1446 \quad 2.1178 \\
& \mathrm{H} \quad 6.4691-3.5859 \quad 2.5152 \\
& \mathrm{H} \quad-5.1324 \quad 2.0767 \quad 2.2221 \\
& \mathrm{H}-4.6625 \quad 3.7805 \quad 2.4291 \\
& \mathrm{H} \quad 6.3386-2.3989 \quad 4.5049 \\
& \text { H } \quad 4.7404-2.0812 \quad 3.6582 \\
& \mathrm{H}-3.1741 \quad 1.4032 \quad 3.7564 \\
& \mathrm{H}-4.6988 \quad 2.1186 \quad 4.4993 \\
& \mathrm{H}-5.5491-3.0719 \quad 1.8397 \\
& \mathrm{H}-3.8470-2.7628 \quad 1.2850 \\
& \text { H } 1.0917 \quad 0.5757 \quad 1.3565 \\
& \begin{array}{llll}
\text { C } & 0.5840 & -0.0392 & 2.1328
\end{array} \\
& \text { C } 0.8129-1.5358 \quad 1.7963 \\
& \mathrm{H} \quad 1.0630 \quad 0.1554 \quad 3.1106 \\
& \begin{array}{llll}
\text { C }-0.8806 & 0.4156 & 2.2085
\end{array} \\
& \text { H } 0.2956-2.1309 \quad 2.5822 \\
& \text { C } 0.2499-1.9770 \quad 0.4419 \\
& \text { C } 2.3269-1.84031 .9578 \\
& \begin{array}{llll}
0 & -1.3988 & 0.5735 & 3.3556
\end{array} \\
& \begin{array}{llll}
0 & -1.4810 & 0.6507 & 1.0970
\end{array} \\
& \text { H } \quad 0.9009-2.7815 \quad 0.0379 \\
& \text { H } 0.2849-1.1608-0.3093 \\
& \begin{array}{llll}
\text { C } & -1.1706 & -2.5423 & 0.4455
\end{array}
\end{aligned}
$$



$\begin{array}{llll}0 & 2.9383 & -2.4012 & 0.9794\end{array}$
○ $2.8740-1.5140 \quad 3.0552$
$\begin{array}{llll}0 & -1.7464 & -2.8362 & 1.5292\end{array}$
$0-1.6903-2.7476-0.7199$
C $-3.6818 \quad 4.6134 \quad 4.8479$
$\begin{array}{llll}\mathrm{H} & -3.0869 & 5.2425 & 5.5354\end{array}$
$\mathrm{H}-3.7694 \quad 5.1240 \quad 3.8642$
$\mathrm{H}-4.6768 \quad 4.4145 \quad 5.2882$
C $-2.6779 \quad 2.6452 \quad 5.9733$
$\mathrm{H}-2.0587 \quad 3.3215 \quad 6.5906$
$\mathrm{H}-3.6325 \quad 2.4348 \quad 6.4887$
$\mathrm{H}-2.1337 \quad 1.7046 \quad 5.7669$
C $-1.6272 \quad 3.5703 \quad 3.9877$
$\mathrm{H}-1.1722 \quad 2.5826 \quad 3.7675$
$\mathrm{H}-1.7929 \quad 4.1507 \quad 3.0665$
$\mathrm{H}-1.0135 \quad 4.1624 \quad 4.6918$
$\begin{array}{llll}\text { C } & 5.2436 & 0.4149 & 2.8648\end{array}$
$\mathrm{H} \quad 5.7529 \quad 0.3480 \quad 1.8906$
$\mathrm{H} \quad 4.2167-0.0046 \quad 2.8111$
$\mathrm{H} \quad 5.2483 \quad 1.4646 \quad 3.2115$
$\begin{array}{llll}\text { C } & 5.4002 & -0.1321 & 5.2274\end{array}$
$\begin{array}{llll}\mathrm{H} & 5.4932 & 0.9466 & 5.4491\end{array}$
H $4.3350-0.4249 \quad 5.1832$
H $5.9312-0.7220 \quad 5.9963$
C $7.4592-0.0065 \quad 3.8583$
$\mathrm{H} \quad 7.8556 \quad-0.1359 \quad 2.8272$
$\mathrm{H} \quad 7.5317 \quad 1.0584 \quad 4.1469$
$\mathrm{H} \quad 8.0163-0.6296 \quad 4.5824$
$\begin{array}{llll}\text { C } & -5.8583 & -5.4766 & 0.9485\end{array}$
$\mathrm{H}-6.4085-5.2536 \quad 1.8807$
$\mathrm{H}-6.4611-5.1699 \quad 0.0751$
$\mathrm{H}-5.6428-6.5588 \quad 0.8901$
$\begin{array}{llll}\text { C }-3.7534 & -5.0543 & 2.1741\end{array}$
$\mathrm{H}-3.5473-6.1402 \quad 2.1762$
$\mathrm{H}-2.8213-4.44752 .1156$
$\mathrm{H}-4.3401-4.7781 \quad 3.0695$
C $-3.7567-5.1124-0.2729$
$\mathrm{H}-4.3808-4.9840-1.1729$
$\mathrm{H}-2.8656-4.4507-0.3488$
$\mathrm{H}-3.4685-6.1734-0.1521$ 

C $3.6168 \quad 3.2059-2.3797$
$\mathrm{H} \quad 2.8867 \quad 3.9444 \quad-2.7689$
$\mathrm{H} \quad 4.3904 \quad 3.7561-1.8070$
H $4.1186 \quad 2.7430 \quad-3.2524$
C $2.0215-2.1982-5.3990$
H $2.2397-3.2454-5.6936$
H $1.2551-1.8019-6.0958$
$\mathrm{H} \quad 2.9421-1.6010-5.5605$
C $-1.6910 \quad 2.5850-4.8010$
$\mathrm{H}-1.3957 \quad 1.9582-5.6665$
$\mathrm{H}-2.6836 \quad 3.0224-5.0279$
$\mathrm{H}-0.9579 \quad 3.4147-4.7283$

Cartesian coordinates of the MARIJ-BP86/SVP optimized structure 1•G3 (E=3378.136710 hartries):
C $-1.3784-0.3482-2.4620$
C $-0.3910-1.3687-2.4712$
C $0.9916-1.0308-2.4573$
C $\quad 1.3818 \quad 0.3345-2.4686$
C $0.3977 \quad 1.3629-2.4600$
C $-0.9797 \quad 1.0180 \quad-2.4731$
C $-2.0346 \quad 2.1166-2.5808$
$\begin{array}{llll}\text { C } \quad 2.8610 & 0.6987 & -2.5724\end{array}$
C $-0.8153-2.8317-2.5775$
C $-2.5504 \quad 1.4362 \quad 2.2097$
C $2.5160 \quad 1.4967 \quad 2.2136$
C $\quad 0.0354-2.9221 \quad 2.2106$
C $\quad 0.8343 \quad 2.8232 \quad-2.5084$
C $2.0375-2.1395-2.5015$
C $-2.8613-0.6999-2.5115$
C $-4.1114 \quad 2.6261-1.3792$
C $4.3365 \quad 2.2471-1.3715$
C $-0.2211-4.8837-1.3716$
C $-5.0659 \quad 3.0108-0.3959$
C $-6.1989 \quad 2.8048-1.3262$
C $-5.1729 \quad 2.5062-2.4353$
C $4.7660 \quad 3.2230-2.4299$
C $5.5332 \quad 3.9671-1.3210$ 

C $5.1428 \quad 2.8863-0.3878$
C $\quad 0.4098 \quad-5.7484 \quad-2.4257$
C $0.6651-6.7819-1.3128$
C $-0.0764-5.8990-0.3845$
C $-3.4173-0.8920-3.9367$
C $2.4846-2.5291-3.9248$
C $-6.0843 \quad 0.3129 \quad 1.1311$
C $\quad 1.2153 \quad 0.7235 \quad 2.2696$
$\begin{array}{llll}\text { C } & 1.2227 & -0.6849 & 2.2744\end{array}$
C $0.0164-1.4090 \quad 2.2683$
$\begin{array}{llll}\text { C } & -1.2070 & -0.7112 & 2.2728\end{array}$
$\begin{array}{llll}\text { C }-1.2310 & 0.6955 & 2.2678\end{array}$
C $-0.0150 \quad 1.4061 \quad 2.2739$
C $-6.1383 \quad 3.4297 \quad 1.7470$
C $\quad 0.1020 \quad-7.0273 \quad 1.7637$
C $6.0311 \quad 3.6172 \quad 1.7561$
C $\quad 1.2098-6.6043 \quad 2.7377$
C $5.1104 \quad 4.3654 \quad 2.7295$
C $-6.3283 \quad 2.2643 \quad 2.7273$
$\begin{array}{lll}\text { C }-7.0385 & 0.0233 & 3.3497\end{array}$
C $-8.3200 \quad 1.2291 \quad 1.6064$
C $3.0983-7.8165 \quad 1.6159$
C $3.5076-6.0948 \quad 3.3484$
C $2.7706-5.4249 \quad 1.1295$
C $5.2033 \quad 6.6050 \quad 1.6014$
C $3.5119 \quad 6.0941 \quad 3.3372$
C $3.3027 \quad 5.1141 \quad 1.1211$
C $0.9503 \quad 3.4002-3.9337$
H $-2.3170 \quad 2.4668-0.4279$
$\begin{array}{llll}\mathrm{H} & 3.3008 & 0.7740 & -0.4185\end{array}$
$\mathrm{H}-0.9748-3.2457-0.4233$
H $0.1313 \quad 3.4427-1.9149$
$\mathrm{H} \quad 1.8082 \quad 2.9323-1.9973$
H $2.9234-1.8413-1.9039$
H $\quad 1.6422-3.0375-1.9923$ 


$$
\begin{aligned}
& \mathrm{H}-3.0473-1.6167-1.9155 \\
& \mathrm{H}-1.8671 \quad-2.8890 \quad-2.9187 \\
& \mathrm{H} \quad 4.6879 \quad 1.9168 \quad 1.3634 \\
& \mathrm{H}-3.9955 \quad 3.1078 \quad 1.3532 \\
& \text { H }-0.6910-5.0126 \quad 1.3619 \\
& \text { H }-6.0313 \quad 4.3259 \quad 2.3995 \\
& \mathrm{H}-7.0300 \quad 3.6056 \quad 1.1113 \\
& \text { H }-0.7260 \quad-7.3782 \quad 2.4209 \\
& \text { H } \quad 0.3927 \quad-7.8913 \quad 1.1321 \\
& \text { H } 6.7526 \quad 3.0812 \quad 2.4136 \\
& \mathrm{H} \quad 6.6297 \quad 4.3009 \quad 1.1203 \\
& \text { Н } \quad 0.9845 \quad-5.5815 \quad 3.1176 \\
& \mathrm{H} \quad 1.2805 \quad-7.3328 \quad 3.5703 \\
& \mathrm{H} \quad 5.7074 \quad 4.7965 \quad 3.5583 \\
& \mathrm{H} \quad 4.3409 \quad 3.6577 \quad 3.1147 \\
& \mathrm{H}-6.9969 \quad 2.5730 \quad 3.5560 \\
& \mathrm{H}-5.3311 \quad 1.9500 \quad 3.1119 \\
& \mathrm{H}-0.2249-3.3592-3.3521 \\
& \mathrm{H} \quad 3.8484 \quad 4.8593 \quad 0.1994 \\
& \text { H } \quad 2.6284 \quad 5.9679 \quad 0.9258 \\
& \mathrm{H} \quad 2.7580 \quad 4.2331 \quad 1.5224 \\
& \mathrm{H} \quad 3.4377-0.1846-2.9089 \\
& \text { H } 2.9147 \quad 6.9575 \quad 2.9920 \\
& \mathrm{H} \quad 4.2080 \quad 6.4116 \quad 4.1343 \\
& \text { H } 2.8462 \quad 5.2907 \quad 3.7028 \\
& \mathrm{H} \quad 3.0243 \quad 1.4707-3.3499 \\
& \text { H } 5.6916 \quad 6.2008 \quad 0.6889 \\
& \text { H } \quad 5.9512 \quad 6.8942 \quad 2.3629 \\
& \mathrm{H} \quad 4.5872 \quad 7.4815 \quad 1.3285 \\
& \text { H }-1.5582 \quad 3.0563-2.9213 \\
& \text { H } 2.2840 \quad-4.5092 \quad 1.5272 \\
& \text { H } \quad 2.2767 \quad-5.7702 \quad 0.2081 \\
& \mathrm{H} \quad 3.8482 \quad-5.2740 \quad 0.9352 \\
& \mathrm{H}-2.7856 \quad 1.8687-3.3560 \\
& \mathrm{H} \quad 3.4294 \quad-6.8544 \quad 4.1472
\end{aligned}
$$




$$
\begin{aligned}
& \text { H } \quad 4.5550 \quad-6.0155 \quad 3.0049 \\
& \text { H } 3.1492-5.1142 \quad 3.7116 \\
& \mathrm{H}-0.0169 \quad 3.3555-4.4747 \\
& \text { H } \quad 2.9687 \quad-8.6065 \quad 2.3789 \\
& \text { H } \quad 2.5051-8.0355 \quad 0.7023 \\
& \text { H } \quad 4.1667-7.7281 \quad 1.3454 \\
& \text { H } 1.2811 \quad 4.4578-3.9044 \\
& \mathrm{H}-8.9408 \quad 1.7418 \quad 2.3646 \\
& \begin{array}{llll}
\mathrm{H} & -8.2106 & 1.8467 & 0.6891
\end{array} \\
& \mathrm{H}-8.7775 \quad 0.2582 \quad 1.3416 \\
& \begin{array}{llll}
\mathrm{H} & -6.1330 & 0.9088 & 0.2065
\end{array} \\
& \mathrm{H}-6.0111-0.1551 \quad 3.7166 \\
& \mathrm{H}-7.6590 \quad 0.4758 \quad 4.1441 \\
& \mathrm{H}-7.4933-0.9250 \quad 3.0105 \\
& \mathrm{H}-0.0293 \quad 2.5050 \quad 2.2648 \\
& \mathrm{H}-6.4936-0.6962 \quad 0.9402 \\
& \begin{array}{llll}
\mathrm{H} & -5.0490 & 0.2767 & 1.5318
\end{array} \\
& \mathrm{H}-2.1517-1.2728 \quad 2.2630 \\
& \mathrm{H} \quad 1.6344 \quad-2.8989-4.5307 \\
& \mathrm{H} \quad 2.9303-1.6690-4.4655 \\
& \mathrm{H} \quad 3.2363-3.3432-3.8920 \\
& \text { H } 2.1814 \quad-1.2222 \quad 2.2655 \\
& \mathrm{H}-4.4985-1.1348-3.9074 \\
& \mathrm{H}-2.8943-1.7089-4.4749 \\
& \mathrm{H}-3.3095 \quad 0.0288-4.5422 \\
& \begin{array}{llll}
\mathrm{H} & -3.4433 & 0.0908 & -2.0038
\end{array} \\
& \mathrm{H} \quad 1.6954 \quad 2.8457-4.5368 \\
& \text { N } 2.6424 \quad-6.5016 \quad 2.1869 \\
& \mathrm{~N} \quad 4.2989 \quad 5.5495 \quad 2.1763 \\
& \begin{array}{llll}
\mathrm{N} & -6.9549 & 0.9691 & 2.1828
\end{array} \\
& \mathrm{~N}-0.3706-5.9490 \quad 0.9214 \\
& \begin{array}{llll}
\mathrm{N} & -4.9657 & 3.2964 & 0.9091
\end{array} \\
& \begin{array}{llll}
\mathrm{N} & 5.3356 & 2.6628 & 0.9189
\end{array} \\
& \mathrm{~N} \quad 3.4775 \quad 1.2202-1.3401 \\
& \mathrm{~N}-0.6777-3.6251-1.3439
\end{aligned}
$$



$\mathrm{N}-2.7928 \quad 2.3943-1.3486$
○ $1.2988-7.8375-1.1600$
$\begin{array}{llll}0-7.4302 & 2.7826 & -1.1760\end{array}$
$\begin{array}{llll}0 & -5.1750 & 2.2021 & -3.6224\end{array}$
$\begin{array}{llll}0 & 4.5078 \quad 3.3728 & -3.6185\end{array}$
$\begin{array}{llll}0 & 1.0141 & -3.5360 & 2.7306\end{array}$
$\begin{array}{llll}0 & -0.9559 & -3.4747 & 1.6065\end{array}$
○ $6.1282 \quad 5.0456-1.1731$
$\begin{array}{llll}0 & 2.5566 & 2.6520 & 2.7324\end{array}$
$\begin{array}{llll}0 & 3.4918 & 0.9145 & 1.6124\end{array}$
$0 \quad 0.6722-5.6039-3.6140$
$\begin{array}{llll}0 & -3.5719 & 0.8973 & 2.7307\end{array}$
$\begin{array}{llll}0 & -2.5324 & 2.5704 & 1.6045\end{array}$

Cartesian coordinates of the MARIJ-BP86/SVP optimized structure 1•G3 (COSMO, $E=-3378.234916$ hartries):
C $-1.3986-0.2673-2.4097$
C $-0.4713-1.3419-2.4210$
C $0.9283-1.0849-2.4072$
C $\quad 1.3954 \quad 0.2554-2.4217$
C $0.4730 \quad 1.3390-2.4117$
C $-0.9212 \quad 1.0729-2.4249$
$\begin{array}{llll}\text { C }-1.9097 & 2.2292 & -2.5392\end{array}$
C $2.8916 \quad 0.5328-2.5333$
C $-0.9796-2.7760-2.5325$
C $-2.4453 \quad 1.6077 \quad 2.1352$
C $2.6209 \quad 1.3191 \quad 2.1392$
C $-0.1616-2.9258 \quad 2.1388$
C $0.9928 \quad 2.7703-2.4637$
C $1.9074-2.2514-2.4543$
C $-2.8985-0.5323-2.4586$
C $-3.9719 \quad 2.8782-1.3882$
C $4.4788 \quad 2.0029-1.3848$
C $-0.5101-4.8862-1.3810$
C $-4.9172 \quad 3.3427-0.4193$
C $-6.01353 .3338-1.4166$
$\begin{array}{llll}\text { C }-4.9848 & 2.9512 & -2.4778\end{array}$
C $5.04532 .8426-2.4770$
C $5.88613 .5508-1.4180$
C $5.3503 \quad 2.5976-0.4174$ 

C $-0.0728-5.8015-2.4718$
C $0.1150-6.8824-1.4106$
C $-0.4364-5.9366-0.4116$
C $-3.4654-0.6909-3.8825$
C $2.3271-2.6652-3.8781$
$\begin{array}{llll}\text { C }-6.3596 & 0.8281 & 1.3594\end{array}$
C $\quad 1.2672 \quad 0.6323 \quad 2.1494$
$\begin{array}{llll}\text { C } & 1.1758 & -0.7724 & 2.1524\end{array}$
C $-0.0789-1.4100 \quad 2.1493$
C $-1.2498-0.6282 \quad 2.1515$
$\begin{array}{llll}\text { C }-1.1745 & 0.7773 & 2.1479\end{array}$
C $0.0878 \quad 1.4004 \quad 2.1512$
$\begin{array}{llll}\text { C }-5.9615 \quad 3.9519 & 1.7128\end{array}$
C $-0.4324-7.1452 \quad 1.7206$
C $6.3978 \quad 3.2119 \quad 1.7117$
$\begin{array}{llll}\text { C } & 0.6616 & -6.9514 & 2.7732\end{array}$
$\begin{array}{llll}\text { C } & 5.6825 \quad 4.0583 & 2.7671\end{array}$
C $-6.3388 \quad 2.9121 \quad 2.7706$
$\begin{array}{llll}\text { C }-7.4504 & 0.8851 & 3.5455\end{array}$
$\begin{array}{llll}\text { C }-8.4388 & 2.1144 & 1.6625\end{array}$
C $2.3961-8.3740 \quad 1.6585$
C $2.9775-6.8999 \quad 3.5356$
C $2.4692-5.9307 \quad 1.3505$
C $6.0287 \quad 6.2749 \quad 1.6519$
$\begin{array}{llll}\text { C } & 4.4709 \quad 6.0299 & 3.5357\end{array}$
C $3.8819 \quad 5.1034 \quad 1.3524$
C $1.1408 \quad 3.3363-3.8893$
$\mathrm{H}-2.2288 \quad 2.5716-0.3879$
$\mathrm{H} \quad 3.3468 \quad 0.6445-0.3816$
$\mathrm{H}-1.1111-3.2219-0.3803$
H $0.3292 \quad 3.4326-1.8746$
H $1.9687 \quad 2.8232-1.9505$
$\mathrm{H} \quad 2.8115-2.0073-1.8628$
$\mathrm{H} \quad 1.4635-3.1222-1.9409$
$\mathrm{H}-3.1395-1.4366-1.8665$
H $-2.0416-2.7719-2.8395$
$\mathrm{H} \quad 4.8353 \quad 1.7052 \quad 1.3379$
$\begin{array}{llll}\mathrm{H} & -3.8811 & 3.3387 & 1.3327\end{array}$
$\mathrm{H}-0.9439-5.0368 \quad 1.3419$
$\mathrm{H}-5.7054 \quad 4.8637 \quad 2.2915$
$\mathrm{H}-6.8142 \quad 4.2246 \quad 1.0629$ 


$$
\begin{aligned}
& \mathrm{H}-1.3474-7.3791 \quad 2.3035 \\
& \mathrm{H}-0.2450-8.0202 \quad 1.0706 \\
& \mathrm{H} \quad 7.0638 \quad 2.5403 \quad 2.2924 \\
& \mathrm{H} \quad 7.0561 \quad 3.8154 \quad 1.0591 \\
& \mathrm{H} \quad 0.5631-5.9467 \quad 3.2280 \\
& \mathrm{H} \quad 0.5560 \quad-7.7251 \quad 3.5546 \\
& \mathrm{H} \quad 6.4072 \quad 4.3583 \quad 3.5448 \\
& \begin{array}{llll}
\mathrm{H} & 4.8672 & 3.4661 & 3.2258
\end{array} \\
& \mathrm{H}-6.9553 \quad 3.3939 \quad 3.5505 \\
& \mathrm{H} \quad-5.4187 \quad 2.4978 \quad 3.2259 \\
& \mathrm{H}-0.4440-3.3191-3.3335 \\
& \text { H } 4.2908 \quad 4.7914 \quad 0.3792 \\
& \text { H } 3.3228 \quad 6.0458 \quad 1.2278 \\
& \mathrm{H} \quad 3.2488 \quad 4.3017 \quad 1.7779 \\
& \mathrm{H} \quad 3.4194-0.3895-2.8385 \\
& \mathrm{H} \quad 3.9965 \quad 6.9795 \quad 3.2383 \\
& \mathrm{H} \quad 5.2954 \quad 6.2211 \quad 4.2421 \\
& \mathrm{H} \quad 3.7248 \quad 5.3592 \quad 3.9933 \\
& \mathrm{H} \quad 3.0943 \quad 1.2666-3.3358 \\
& \mathrm{H} \quad 6.3793 \quad 5.8180 \quad 0.7086 \\
& \mathrm{H} \quad 6.8701 \quad 6.4358 \quad 2.3468 \\
& \mathrm{H} \quad 5.5307 \quad 7.2345 \quad 1.4338 \\
& \mathrm{H}-1.3760 \quad 3.1468-2.8479 \\
& \text { H } 2.0988-4.9781 \quad 1.7740 \\
& \mathrm{H} \quad 1.9906-6.1291 \quad 0.3793 \\
& \mathrm{H} \quad 3.5645 \quad-5.9250 \quad 1.2225 \\
& \mathrm{H}-2.6479 \quad 2.0349-3.3392 \\
& \mathrm{H} \quad 2.7285-7.7064 \quad 4.2445 \\
& \mathrm{H} \quad 4.0360 \quad-6.9704 \quad 3.2354 \\
& \text { H } 2.7765 \quad-5.9161 \quad 3.9914 \\
& \text { H } 0.1726 \quad 3.3505-4.4273 \\
& \mathrm{H} \quad 2.1120 \quad-9.1792 \quad 2.3570 \\
& \begin{array}{llll}
\mathrm{H} & 1.8221 & -8.4482 & 0.7172
\end{array} \\
& \mathrm{H} \quad 3.4752-8.4302 \quad 1.4374 \\
& \mathrm{H} \quad 1.5289 \quad 4.3732 \quad-3.8594 \\
& \mathrm{H}-8.9935 \quad 2.7669 \quad 2.3577 \\
& \begin{array}{llll}
\mathrm{H} & -8.2177 & 2.6437 & 0.7178
\end{array} \\
& \mathrm{H}-9.0270 \quad 1.2064 \quad 1.4475 \\
& \mathrm{H}-6.2956 \quad 1.3354 \quad 0.3845 \\
& \mathrm{H}-6.4973 \quad 0.5697 \quad 4.0015 \\
& \mathrm{H}-8.0232 \quad 1.5082 \quad 4.2519
\end{aligned}
$$




$$
\begin{array}{lrrr}
\text { H } & -8.0414 & 0.0022 & 3.2512 \\
\mathrm{H} & 0.1547 & 2.4964 & 2.1447 \\
\mathrm{H} & -6.9012 & -0.1250 & 1.2392 \\
\mathrm{H} & -5.3482 & 0.6764 & 1.7818 \\
\mathrm{H} & -2.2324 & -1.1188 & 2.1455 \\
\mathrm{H} & 1.4551 & -2.9761 & -4.4852 \\
\mathrm{H} & 2.8255 & -1.8348 & -4.4164 \\
\mathrm{H} & 3.0303 & -3.5203 & -3.8453 \\
\mathrm{H} & 2.0920 & -1.3779 & 2.1470 \\
\mathrm{H} & -4.5573 & -0.8741 & -3.8503 \\
\mathrm{H} & -2.9942 & -1.5378 & -4.4190 \\
\mathrm{H} & -3.2994 & 0.2190 & -4.4905 \\
\mathrm{H} & -3.4313 & 0.2878 & -1.9466 \\
\mathrm{H} & 1.8465 & 2.7354 & -4.4946 \\
\mathrm{~N} & 2.1172 & -7.0448 & 2.3068 \\
\mathrm{~N} & 5.0255 & 5.3620 & 2.3038 \\
\mathrm{~N} & -7.1474 & 1.6954 & 2.3117 \\
\mathrm{~N} & -0.6798 & -5.9737 & 0.8963 \\
\mathrm{~N} & -4.8252 & 3.5766 & 0.8876 \\
\mathrm{~N} & 5.5088 & 2.4068 & 0.8902 \\
\mathrm{~N} & 3.5365 & 1.0580 & -1.3184 \\
\mathrm{~N} & -0.8492 & -3.5955 & -1.3165 \\
\mathrm{~N} & -2.6843 & 2.5274 & -1.3235 \\
\mathrm{O} & 0.5546 & -8.0418 & -1.3585 \\
\mathrm{O} & -7.2372 & 3.5325 & -1.3636 \\
\mathrm{O} & -4.9802 & 2.7264 & -3.6926 \\
\mathrm{O} & 4.8495 & 2.9449 & -3.6926 \\
\mathrm{O} & 0.7530 & -3.5835 & 2.6994 \\
\mathrm{O} & -1.1857 & -3.4277 & 1.5373 \\
\mathrm{O} & 6.6647 & 4.5158 & -1.3682 \\
\mathrm{O} & 2.7330 & 2.4394 & 2.7009 \\
\mathrm{O} & 3.5678 & 0.6843 & 1.5365 \\
\mathrm{O} & 0.1131 & -5.6868 & -3.6878 \\
\mathrm{O} & -3.4722 & 1.1473 & 2.6980 \\
\mathrm{O} & -2.3669 & 2.7437 & 1.5301
\end{array}
$$

Cartesian coordinates of the MARIJ-BP86/SVP optimized structure 1•G4 (E=3077.041778 hartries):
C $-0.2246-0.2058 \quad 0.1825$
C $1.1920-0.1078 \quad 0.2825$
C $\quad 1.8235 \quad 1.1614 \quad 0.3808$ 

C $\quad 1.0371 \quad 2.3333 \quad 0.2482$
C $-0.3541 \quad 2.2487-0.0233$
C $-0.9900 \quad 0.9795 \quad 0.0080$
$\begin{array}{lll}\text { C }-2.5102 & 0.9482 & 0.0116\end{array}$
C $\quad 1.5878 \quad 3.6976 \quad 0.6356$
C $2.0342-1.3710 \quad 0.3699$
$\mathrm{N}-2.9865 \quad 1.6115 \quad 1.2413$
$\begin{array}{llll}\mathrm{N} & 1.1017 & 3.9479 & 2.0097\end{array}$
N $2.3903-1.7079 \quad 1.7646$
C $-1.1332 \quad 3.5070-0.3966$
C $\quad 3.3298 \quad 1.2654 \quad 0.5741$
C $-0.9116-1.56690 .2258$
C $-4.2157 \quad 2.1252 \quad 1.3980$
C $\quad 1.6332 \quad 4.7915 \quad 2.9007$
C $3.5433-2.3112 \quad 2.0902$
$\begin{array}{lll}\text { C }-4.7790 & 2.9728 & 2.3817\end{array}$
C $-6.1384 \quad 2.8498 \quad 1.7974$
$\begin{array}{llll}\text { C }-5.5083 & 2.0416 & 0.6348\end{array}$
C $2.7920 \quad 5.7489 \quad 2.9212$
C $2.4158 \quad 6.0372 \quad 4.3974$
C $1.3359 \quad 5.0290 \quad 4.2636$
C $4.7555-2.6438 \quad 1.2649$
C $5.3860 \quad-3.2021 \quad 2.5531$
C $4.1546-2.7875 \quad 3.2803$
$\begin{array}{llll}0 & -5.8553 & 1.5231 & -0.4114\end{array}$
$\begin{array}{llll}0 & -7.2598 & 3.2005 & 2.1771\end{array}$
$\begin{array}{llll}0 & 5.0731 & -2.4519 & 0.1025\end{array}$
$\begin{array}{llll}0 & 6.4627 & -3.6859 & 2.9117\end{array}$
○ $3.6458 \quad 6.0793 \quad 2.1185$
$\begin{array}{llll}0 & 2.8430 \quad 6.7185 & 5.3337\end{array}$
$\mathrm{N} \quad 0.4262 \quad 4.4870 \quad 5.1028$
$\begin{array}{llll}\mathrm{N} & -4.2866 \quad 3.5693 & 3.4814\end{array}$
N $\quad 3.8189 \quad-2.8577 \quad 4.5868$
C $-5.1377 \quad 4.3217 \quad 4.3823$
C $4.6719-3.5804 \quad 5.5212$ 

C $0.2797 \quad 4.9076 \quad 6.4858$
C $5.2690-2.7200 \quad 6.6370$
C $0.8661 \quad 3.9713 \quad 7.5618$
$\begin{array}{lll}\text { C }-5.3347 & 3.6709 & 5.7574\end{array}$
$\begin{array}{llll}\mathrm{N} & -6.3217 & 2.4974 & 5.8521\end{array}$
N $2.3437 \quad 4.1410 \quad 7.9466$
$\begin{array}{llll}\mathrm{N} & 6.4139 & -1.7671 & 6.2627\end{array}$
C $2.6596 \quad 5.5705 \quad 8.3008$
C $2.5826 \quad 3.2412 \quad 9.1340$
C $3.2558 \quad 3.6749 \quad 6.8349$
$\begin{array}{llll}\text { C } \quad 5.9547 & -0.7260 \quad 5.2624\end{array}$
C $6.8155-1.0554 \quad 7.5262$
C $7.5932-2.5256 \quad 5.7079$
C $-7.6960 \quad 2.9013 \quad 5.3790$
C $-6.3753 \quad 2.0953 \quad 7.3017$
C $-5.8416 \quad 1.3024 \quad 5.0564$
C $4.0916 \quad 1.5215-0.7405$
C $-1.1999-2.1641-1.1649$
C $-0.9478 \quad 3.8710-1.8839$
$\mathrm{H}-2.9031-0.0845-0.0401$
Н $-2.9368 \quad 1.4740 \quad-0.8669$
$\mathrm{H} \quad 1.2398 \quad 4.4977-0.0490$
H $\quad 2.6911 \quad 3.7482 \quad 0.6329$
H $\quad 2.9847-1.2772 \quad-0.1886$
H $\quad 1.5008-2.2253-0.0920$
$\mathrm{H}-2.2863 \quad 1.8322 \quad 1.9649$
H $\quad 0.3061 \quad 3.3727 \quad 2.3180$
H $1.6713-1.5678 \quad 2.4797$
$\mathrm{H}-0.8258 \quad 4.3635 \quad 0.2357$
$\mathrm{H}-2.2096 \quad 3.3800 \quad-0.1893$
$\mathrm{H} \quad 3.5577 \quad 2.0756 \quad 1.2964$
$\mathrm{H} \quad 3.7195 \quad 0.3440 \quad 1.0441$
$\mathrm{H}-1.8617-1.48390 .7919$
$\begin{array}{llll}\mathrm{H} & -0.3019 & -2.2844 & 0.8097\end{array}$
$\mathrm{H}-0.3106 \quad 3.8619 \quad 4.7023$ 


$$
\begin{aligned}
& \mathrm{H}-3.2763 \quad 3.3963 \quad 3.7476 \\
& \mathrm{H} \quad 3.1837-2.1446 \quad 5.0369 \\
& \mathrm{H}-6.0990 \quad 4.5384 \quad 3.8753 \\
& \mathrm{H}-4.6709 \quad 5.3084 \quad 4.6018 \\
& \mathrm{H} \quad 5.4460-4.1320 \quad 4.9522 \\
& \text { H } \quad 4.0702-4.3479 \quad 6.0596 \\
& \mathrm{H}-0.8056 \quad 4.9524 \quad 6.7151 \\
& \mathrm{H} \quad 0.6626 \quad 5.9414 \quad 6.5986 \\
& \mathrm{H} \quad 5.6811 \quad-3.3774 \quad 7.4279 \\
& \text { H } \quad 4.4735-2.0706 \quad 7.0568 \\
& \mathrm{H} \quad 0.7664 \quad 2.9007 \quad 7.2822 \\
& \mathrm{H} \quad 0.3158 \quad 4.1429 \quad 8.5082 \\
& \mathrm{H} \quad-5.7171 \quad 4.4252 \quad 6.4734 \\
& \mathrm{H}-4.3615 \quad 3.2595 \quad 6.1012 \\
& \mathrm{H} \quad 3.6737 \quad 5.6000 \quad 8.7388 \\
& \begin{array}{llll}
\mathrm{H} & 2.6431 & 6.1832 & 7.3754
\end{array} \\
& \mathrm{H} \quad 1.9245 \quad 5.9289 \quad 9.0450 \\
& \mathrm{H} \quad 3.6437 \quad 3.3298 \quad 9.4293 \\
& \text { H } \quad 1.9357 \quad 3.5652 \quad 9.9689 \\
& \mathrm{H} \quad 2.3507 \quad 2.2053 \quad 8.8168 \\
& \mathrm{H} \quad 3.0250 \quad 2.6043 \quad 6.6507 \\
& \mathrm{H} \quad 3.0901 \quad 4.3052 \quad 5.9486 \\
& \mathrm{H} \quad 4.2979 \quad 3.8080 \quad 7.1794 \\
& \mathrm{H} \quad 5.8842 \quad-1.1959 \quad 4.2682 \\
& \mathrm{H} \quad 4.9574-0.3557 \quad 5.5822 \\
& \mathrm{H} \quad 6.7134 \quad 0.0775 \quad 5.2340 \\
& \text { H } \quad 7.6538-0.3719 \quad 7.3016 \\
& \mathrm{H} \quad 5.9466 \quad-0.4844 \quad 7.8984 \\
& \text { H } \quad 7.1308-1.7992 \quad 8.2794 \\
& \mathrm{H} \quad 7.2982-3.0206 \quad 4.7585 \\
& \mathrm{H} \quad 8.4088-1.8057 \quad 5.5137 \\
& \mathrm{H} \quad 7.9220 \quad-3.2709 \quad 6.4551 \\
& \mathrm{H}-8.0234 \quad 3.7907 \quad 5.9484 \\
& \mathrm{H}-7.6611 \quad 3.1143 \quad 4.2890 \\
& \begin{array}{llll}
\mathrm{H} & -8.3889 & 2.0613 & 5.5678
\end{array}
\end{aligned}
$$




$$
\begin{aligned}
& \mathrm{H}-7.0794 \quad 1.2514 \quad 7.4139 \\
& \mathrm{H}-5.3623 \quad 1.7886 \quad 7.6184 \\
& \mathrm{H}-6.7215 \quad 2.9536 \quad 7.9051 \\
& \mathrm{H}-5.9523 \quad 1.5189 \quad 3.9820 \\
& \mathrm{H}-4.7776 \quad 1.1254 \quad 5.3086 \\
& \begin{array}{llll}
\mathrm{H} & -6.4775 & 0.4391 & 5.3242
\end{array} \\
& \text { H } 3.7735 \quad 2.4698-1.2187 \\
& \text { H } 5.1833 \quad 1.5792-0.5592 \\
& \text { H } 3.9156 \quad 0.7079-1.4719 \\
& \mathrm{H}-1.7021-3.1488-1.0797 \\
& \text { H }-1.8551-1.4995-1.7631 \\
& \text { H }-0.2658-2.3060-1.7453 \\
& \text { H }-1.2983 \quad 3.0507-2.5421 \\
& \mathrm{H}-1.5250 \quad 4.7822 \quad-2.1394 \\
& \text { H } \quad 0.1161 \quad 4.0597-2.1332 \\
& \text { C } 0.5393-0.9355 \quad 6.4110 \\
& \text { C }-0.6901-0.0273 \quad 6.3097 \\
& \text { C } \quad 1.8858-0.1895 \quad 6.5509 \\
& \text { C }-0.6904 \quad 0.8325 \quad 5.0433 \\
& \begin{array}{llll}
0 & 1.8856 & 0.9549 & 7.0863
\end{array} \\
& \text { ○ } 2.9306-0.8019 \quad 6.1339 \\
& \text { C }-1.8106 \quad 1.8786 \quad 4.9533 \\
& \begin{array}{llll}
0 & -2.7777 & 1.8252 & 5.7516
\end{array} \\
& \begin{array}{llll}
0 & -1.6687 & 2.7830 & 4.0280
\end{array} \\
& \mathrm{H} \quad 0.6037-1.6265 \quad 5.5426 \\
& \text { H } \quad 0.4486-1.5926 \quad 7.3049 \\
& \mathrm{H}-1.6193-0.6325 \quad 6.3490 \\
& \begin{array}{llll}
\mathrm{H} & -0.7201 & 0.6354 & 7.1987
\end{array} \\
& \mathrm{H}-0.7673 \quad 0.1957 \quad 4.1321 \\
& \mathrm{H} \quad 0.2751 \quad 1.3721 \quad 4.9396
\end{aligned}
$$

Cartesian coordinates of the MARIJ-BP86/SVP optimized structure 1•G5 (E=3037.753785 hartries):
C $\quad 1.3316-0.4926-3.4469$
C $2.3891 \quad 0.3475-3.0023$
C $2.1128 \quad 1.6351 \quad-2.4674$ 

C $0.7860 \quad 2.1346-2.5248$
C $-0.2465 \quad 1.3764-3.1396$
C $0.0175 \quad 0.0367-3.5280$
C $-1.1691-0.8423-3.8823$
C $0.3871 \quad 3.4099-1.8010$
C $3.8150-0.1862-2.9923$
$\mathrm{N}-1.9784-1.0423-2.6590$
$\mathrm{N}-0.4985 \quad 3.0296-0.6776$
N $4.1485-0.8037-1.7032$
C $-1.5965 \quad 2.0169-3.4573$
C $3.2353 \quad 2.4866-1.8918$
C $1.6103-1.9338-3.8574$
C $-3.1152-1.7422-2.6013$
C $-1.0184 \quad 3.8726 \quad 0.2208$
C $5.3226-0.6228-1.0878$
C $-4.1099-1.8853-1.6040$
C $-4.8519-2.9000 \quad-2.3838$
C $-3.8367-2.6839-3.5343$
C $-0.7343 \quad 5.2909 \quad 0.6521$
C $-1.8285 \quad 5.0839 \quad 1.7329$
C $-2.0730 \quad 3.7440 \quad 1.1546$
C $6.5298 \quad 0.2345-1.3308$
C $\quad 7.0644-0.2726 \quad 0.0290$
C $5.8487-1.1133 \quad 0.1354$
$\begin{array}{llll}0 & -3.6419 & -3.0871 & -4.6644\end{array}$
$\begin{array}{llll}0 & -5.8234 & -3.6225 & -2.1331\end{array}$
○ $6.8400 \quad 1.0472-2.1907$
$\begin{array}{llll}0 & 7.9977 & -0.0253 & 0.8028\end{array}$
$\begin{array}{llll}0 & 0.0481 & 6.1521 & 0.3004\end{array}$
$\begin{array}{llll}0 & -2.2931 & 5.6859 & 2.7066\end{array}$
$\begin{array}{llll}\mathrm{N}-2.9382 & 2.7509 & 1.4627\end{array}$
$\mathrm{N}-4.2729-1.3635-0.3674$
N $5.3347-1.9323 \quad 1.0683$
$\begin{array}{llll}\text { C }-5.4625 & -1.6285 & 0.4178\end{array}$
C $6.0013-2.1897 \quad 2.3251$ 

C $-4.0658 \quad 2.9841 \quad 2.3461$
C $5.3211-1.5442 \quad 3.5404$
C $-4.0906 \quad 2.1013 \quad 3.5965$
C $-5.2232-2.4230 \quad 1.7073$
$\mathrm{N}-4.9996-3.9363 \quad 1.5724$
$\begin{array}{llll}\mathrm{N} & -3.1089 & 2.4560 & 4.7225\end{array}$
$\mathrm{N} \quad 5.5616 \quad-0.0438 \quad 3.7741$
H $-0.8653-1.8253-4.2838$
$\mathrm{H}-1.8026-0.3761-4.6656$
H $-0.1519 \quad 4.1115-2.4733$
$\mathrm{H} \quad 1.2542 \quad 3.9681-1.4062$
$\mathrm{H} \quad 4.5580 \quad 0.6109-3.1842$
$\mathrm{H} \quad 3.9488-0.9250 \quad-3.8092$
H $-1.6862-0.5293-1.8143$
$\mathrm{H}-0.8419 \quad 2.0593-0.6709$
H $3.4193-1.3288-1.1750$
$\mathrm{H}-1.8475 \quad 2.8036 \quad-2.7217$
$\mathrm{H}-2.4166 \quad 1.2788-3.3688$
H $2.8840 \quad 2.9929-0.9690$
H $\quad 4.0742 \quad 1.8448-1.5673$
$\mathrm{H} \quad 0.7756-2.5812 \quad-3.5192$
$\mathrm{H} \quad 2.4947 \quad-2.3194 \quad-3.3144$
$\begin{array}{llll}\mathrm{H} & -2.8308 & 1.8202 & 0.9987\end{array}$
$\mathrm{H}-3.5779-0.6575-0.0218$
H $4.2933-2.1449 \quad 0.9576$
H $-6.2215-2.1111-0.2297$
$\mathrm{H}-5.9099-0.6689 \quad 0.7607$
H $\quad 7.0707-1.9107 \quad 2.2401$
$\mathrm{H} \quad 5.9804 \quad-3.2817 \quad 2.5427$
$\mathrm{H}-5.0206 \quad 2.7561 \quad 1.8185$
$\mathrm{H}-4.1019 \quad 4.0636 \quad 2.5926$
H $\quad 5.6722 \quad-2.0380 \quad 4.4687$
H $4.2176-1.6435 \quad 3.4389$
$\mathrm{H}-3.8519 \quad 1.0532 \quad 3.3044$
$\mathrm{H}-5.0947 \quad 2.1510 \quad 4.0628$ 


$$
\begin{aligned}
& \text { H }-6.1098-2.3265 \quad 2.3647 \\
& \begin{array}{llll}
\mathrm{H} & -4.3331 & -2.0065 & 2.2285
\end{array} \\
& \text { H } \quad 0.2263 \quad-1.8509 \quad 0.0982 \\
& \begin{array}{llll}
\text { C } & 0.5495 & -1.0385 & 0.7802
\end{array} \\
& \begin{array}{llll}
\mathrm{H} & 0.6147 & -0.1247 & 0.1494
\end{array} \\
& \text { C }-0.4383-0.8179 \quad 1.9220 \\
& \begin{array}{llll}
\text { C } & 1.9825 & -1.3594 & 1.2705
\end{array} \\
& \begin{array}{llll}
\mathrm{H} & 0.0120 & -0.0714 & 2.6162
\end{array} \\
& \mathrm{H}-0.5382-1.7296 \quad 2.5474 \\
& \begin{array}{llll}
\text { C }-1.8545 & -0.3440 & 1.5842
\end{array} \\
& \begin{array}{llll}
0 & 2.3241 & -0.9579 & 2.4212
\end{array} \\
& \begin{array}{llll}
0 & 2.7209 & -2.0038 & 0.4496
\end{array} \\
& \begin{array}{llll}
0 & -2.0881 & 0.2231 & 0.4467
\end{array} \\
& \begin{array}{llll}
0 & -2.7472 & -0.5058 & 2.4741
\end{array} \\
& \text { C }-3.2908 \quad 3.8835 \quad 5.1789 \\
& \mathrm{H}-2.6437 \quad 4.0508 \quad 6.0589 \\
& \mathrm{H}-2.9965 \quad 4.5769 \quad 4.3596 \\
& \mathrm{H}-4.3491 \quad 4.0336 \quad 5.4609 \\
& \text { C }-3.3916 \quad 1.5121 \quad 5.8600 \\
& \begin{array}{llll}
\mathrm{H} & -2.6869 & 1.7211 & 6.6849
\end{array} \\
& \mathrm{H}-4.4292 \quad 1.6623 \quad 6.2082 \\
& \begin{array}{llll}
\mathrm{H} & -3.2563 & 0.4777 & 5.4956
\end{array} \\
& \begin{array}{lll}
\text { C }-1.6782 & 2.2428 & 4.2871
\end{array} \\
& \mathrm{H}-1.5912 \quad 1.2168 \quad 3.8851 \\
& \begin{array}{llll}
\mathrm{H} & -1.4211 & 2.9831 & 3.5136
\end{array} \\
& \begin{array}{llll}
\mathrm{H} & -1.0278 & 2.3809 & 5.1697
\end{array} \\
& \begin{array}{llll}
\text { C } & 4.9220 & 0.7922 & 2.6862
\end{array} \\
& \begin{array}{llll}
\mathrm{H} & 5.4987 & 0.6688 & 1.7557
\end{array} \\
& \begin{array}{llll}
\mathrm{H} & 3.8804 & 0.4331 & 2.5489
\end{array} \\
& \mathrm{H} \quad 4.9626 \quad 1.8500 \quad 3.0043 \\
& \text { C } 4.8885 \quad 0.3004 \quad 5.0736 \\
& \mathrm{H} \quad 5.0260 \quad 1.3785 \quad 5.2737 \\
& \text { H } 3.8133 \quad 0.0635 \quad 4.9815 \\
& \text { H } 5.3423-0.2918 \quad 5.8886 \\
& \begin{array}{llll}
\text { C } \quad 7.0344 & 0.2698 \quad 3.8568
\end{array}
\end{aligned}
$$




$$
\begin{aligned}
& \begin{array}{llll}
\mathrm{H} & 7.5011 & 0.1117 & 2.8592
\end{array} \\
& \text { H } 7.1467 \quad 1.3300 \quad 4.1487 \\
& \mathrm{H} \quad 7.4963-0.3799 \quad 4.6231 \\
& \text { C }-6.1580-4.5961 \quad 0.8593 \\
& \mathrm{H}-7.0938-4.3250 \quad 1.3810 \\
& \mathrm{H}-6.1756-4.2622-0.2022 \\
& \mathrm{H}-6.0107-5.6904 \quad 0.9015 \\
& \text { C }-4.8840-4.4922 \quad 2.9667 \\
& \mathrm{H}-4.7034-5.5804 \quad 2.9059 \\
& \mathrm{H}-4.0418-3.9960 \quad 3.4812 \\
& \mathrm{H}-5.8251-4.3006 \quad 3.5122 \\
& \begin{array}{llll}
\text { C } & -3.7211 & -4.2445 & 0.8297
\end{array} \\
& \mathrm{H}-3.8258-3.9321-0.2210 \\
& \mathrm{H}-2.8895 \quad-3.6994 \quad 1.3093 \\
& \mathrm{H}-3.5479 \quad-5.3349 \quad 0.8774 \\
& \text { C } 3.7719 \quad 3.5380 \quad-2.8818 \\
& \mathrm{H} \quad 2.9828 \quad 4.2545 \quad-3.1881 \\
& \mathrm{H} \quad 4.6040 \quad 4.1134-2.4302 \\
& \mathrm{H} \quad 4.1606 \quad 3.0582-3.8022 \\
& \begin{array}{llll}
\text { C } & 1.8194 & -2.1219 & -5.3724
\end{array} \\
& \mathrm{H} \quad 2.0261-3.1836-5.6150 \\
& \text { H } \quad 0.9257-1.8098 \quad-5.9501 \\
& \text { H } \quad 2.6722-1.5169-5.7417 \\
& \text { C }-1.6129 \quad 2.6304 \quad-4.8729 \\
& \mathrm{H}-1.4141 \quad 1.8653-5.6498 \\
& \mathrm{H}-2.5977 \quad 3.0904-5.0912 \\
& \mathrm{H}-0.8372 \quad 3.4151-4.9809
\end{aligned}
$$


ITC curve and thermodynamic data of $1 \cdot \mathrm{G} 1 \mathrm{1:3} \mathrm{v} / \mathrm{v} \mathrm{H}_{2} \mathrm{O} / \mathrm{EtOH}$ :

See Figure 5 (top) of the manuscript.

ITC curve and thermodynamic data of $1 \cdot \mathrm{G} 11: 1 \mathrm{v} / \mathrm{v} \mathrm{H}_{2} \mathrm{O} / \mathrm{EtOH}$
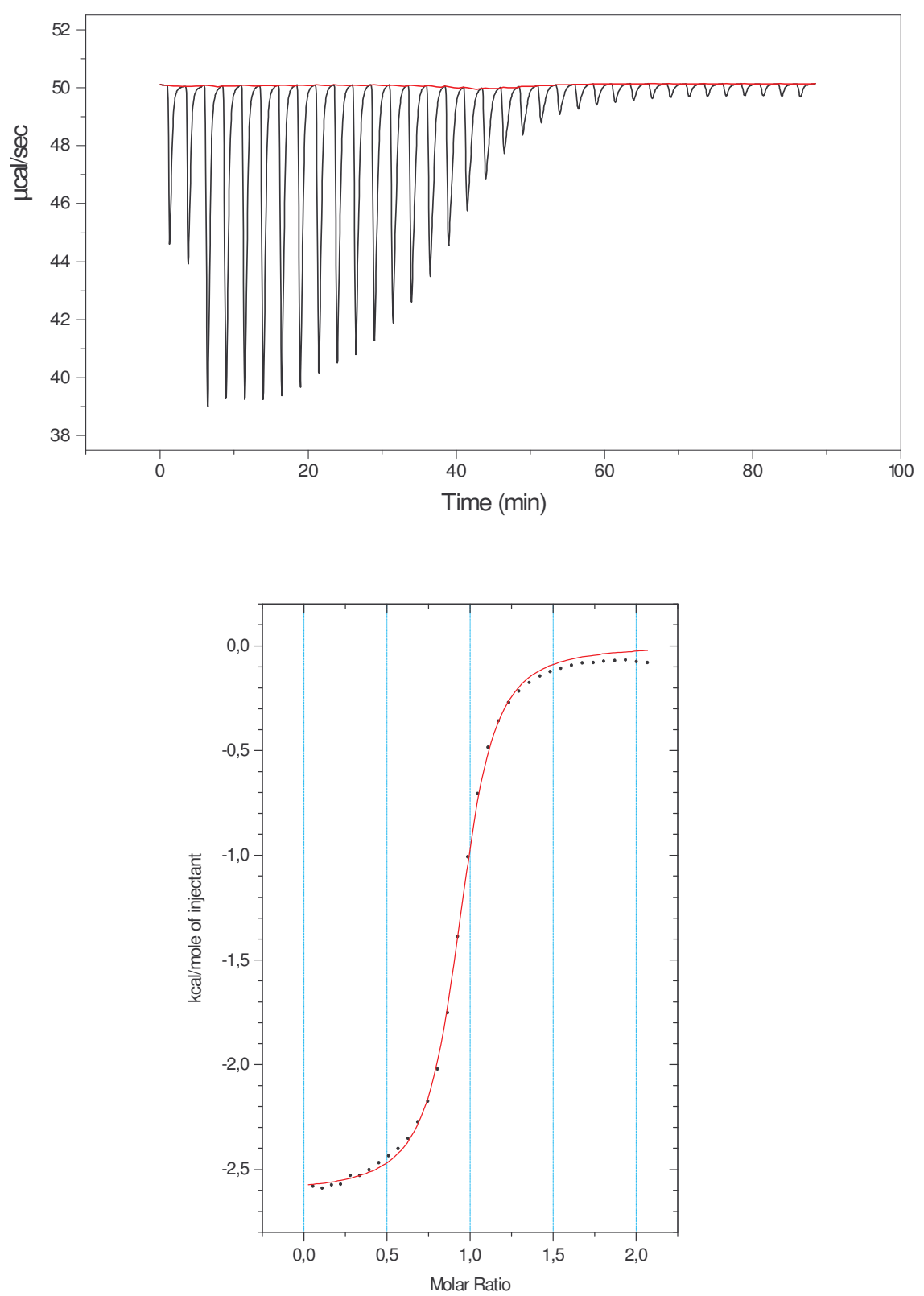

Figure S1. 
ITC curve and thermodynamic data of $1 \cdot \mathrm{G} 1$ 1:3 v/v $\mathrm{H}_{2} \mathrm{O} / \mathrm{EtOH}$ (sodium salt):
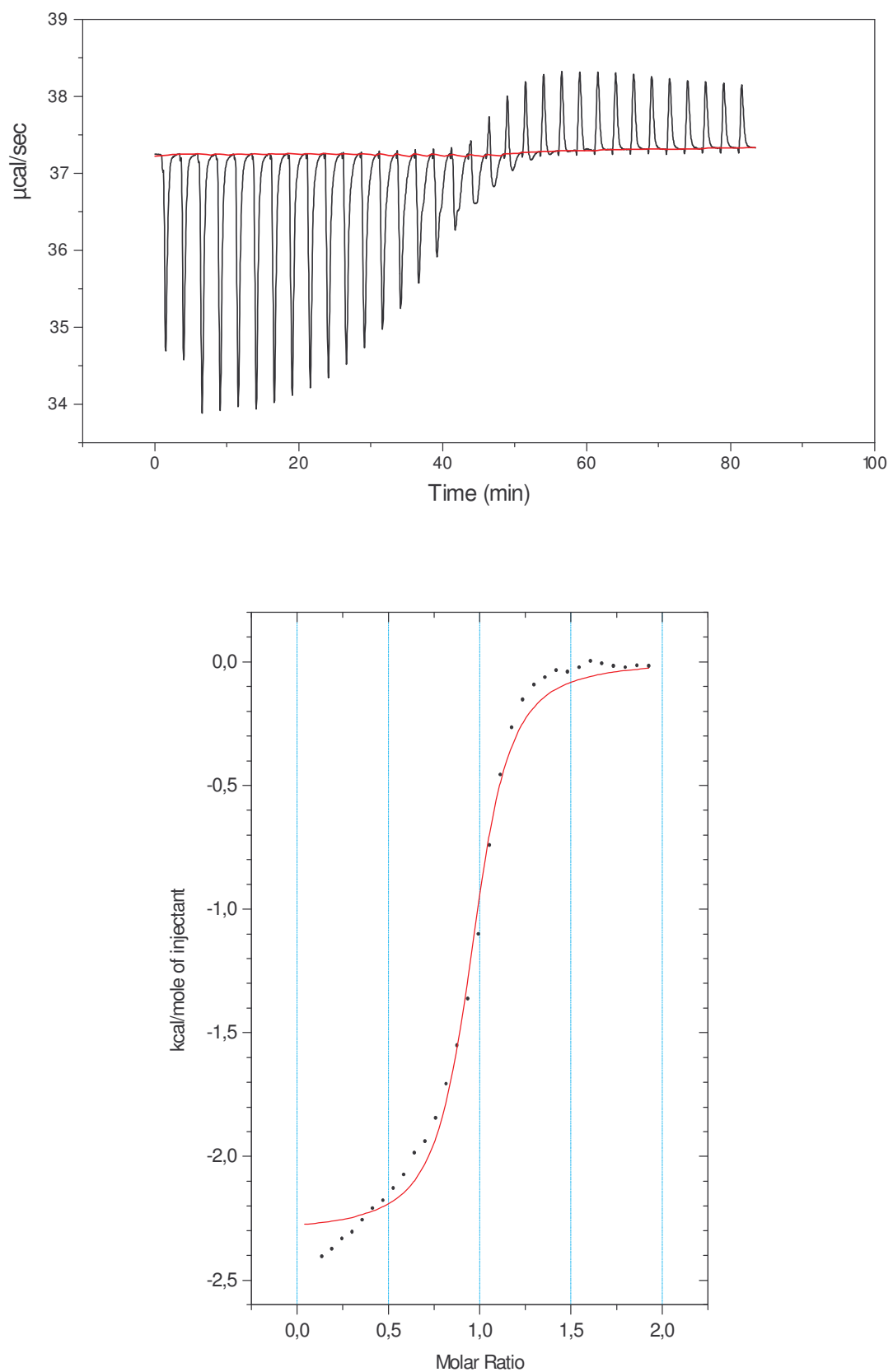

Figure S2. 
ITC curve and thermodynamic data of $1 \cdot \mathrm{G} 21: 3 \mathrm{v} / \mathrm{v} \mathrm{H} \mathrm{H}_{2} \mathrm{O} / \mathrm{EtOH}$ :
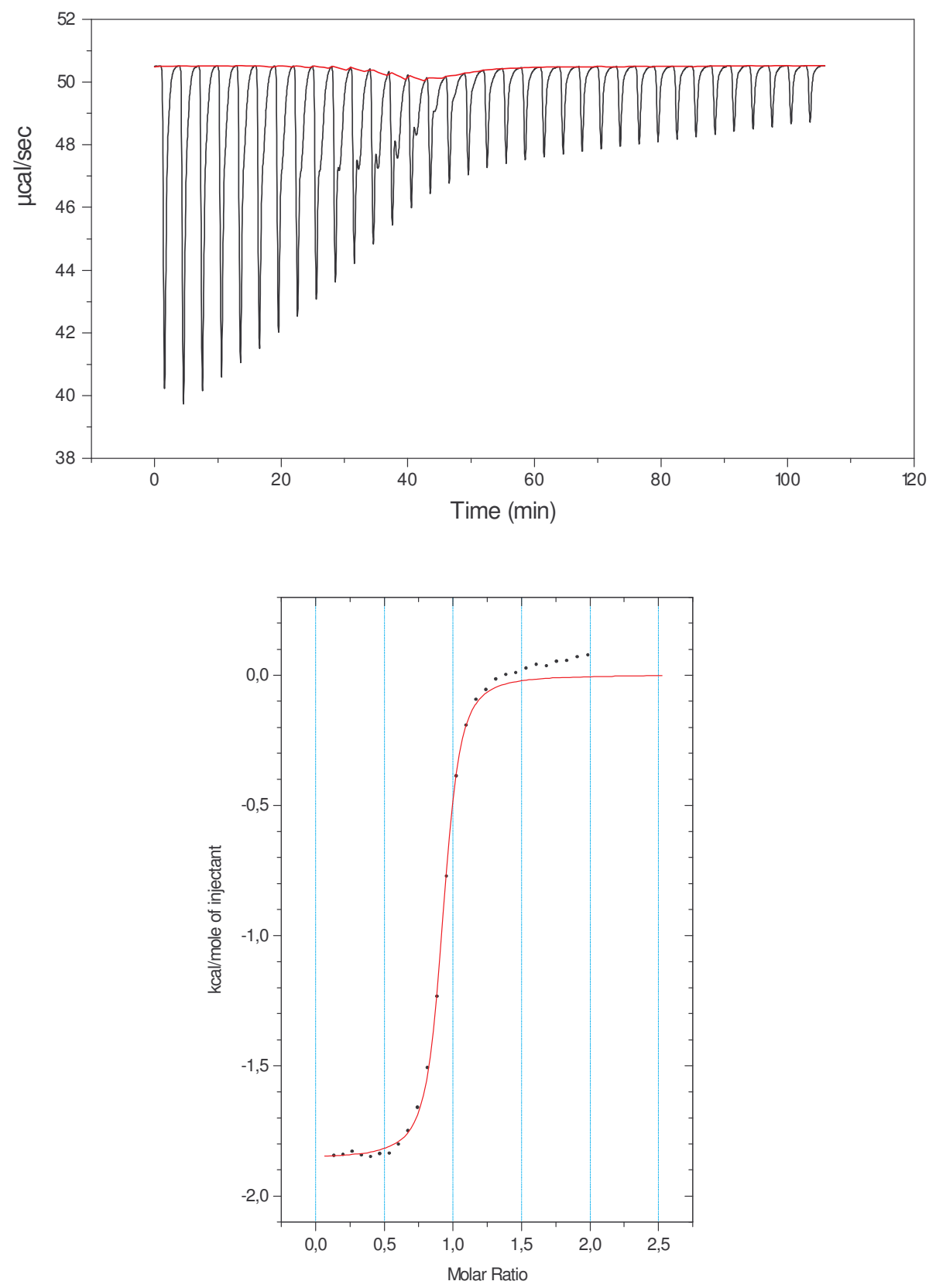

Figure S3.

ITC curve and thermodynamic data of $1 \cdot \mathrm{G3} 1: 3 \mathrm{v} / \mathrm{v} \mathrm{H} \mathrm{H}_{2} \mathrm{O} / \mathrm{EtOH}$ :

See Figure 5 (bottom) of the manuscript. 
ITC curve and thermodynamic data of $1 \cdot G 41: 3 \mathrm{v} / \mathrm{v} \mathrm{H}_{2} \mathrm{O} / \mathrm{EtOH}$ :
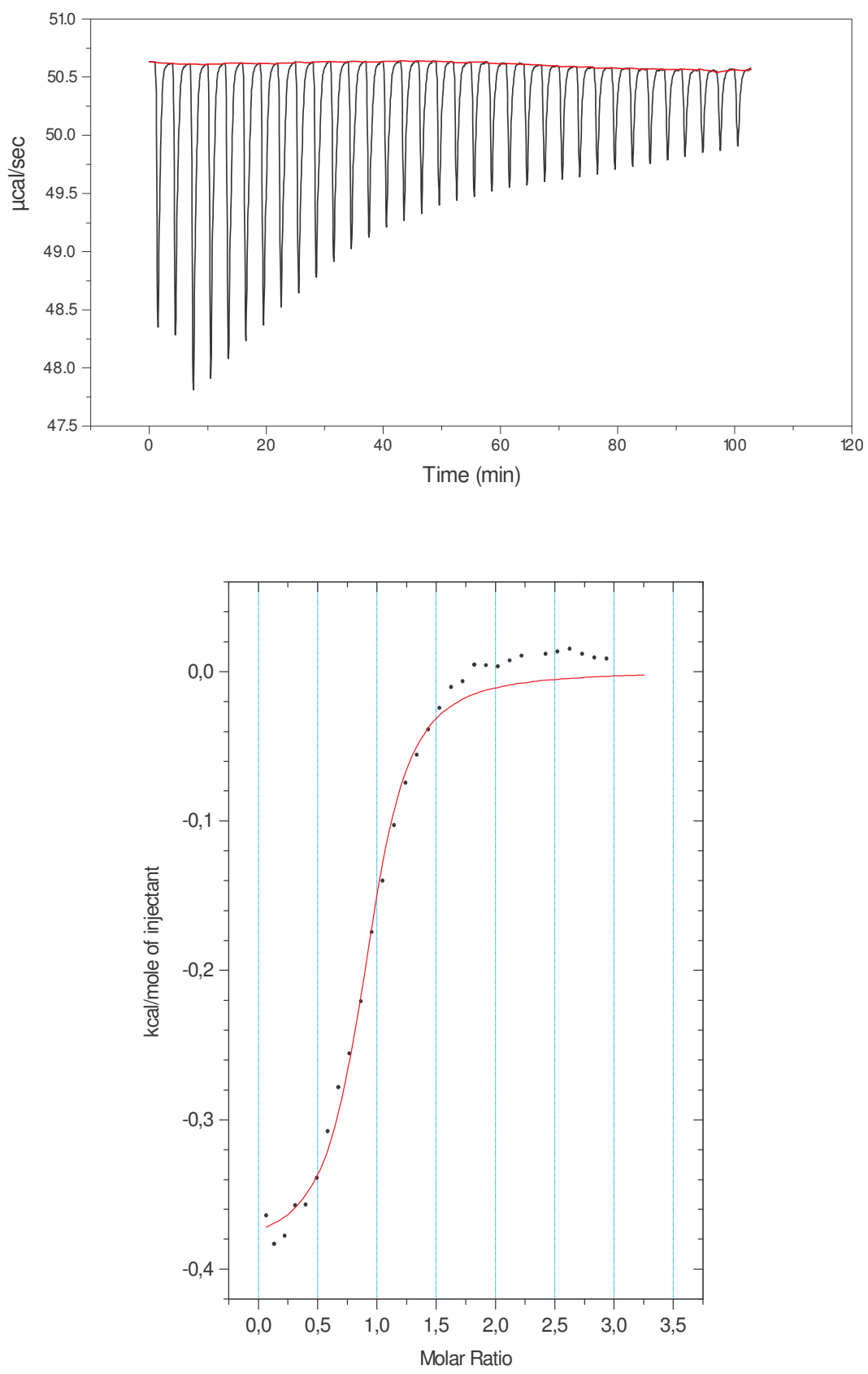

Figure S4 
ITC curve and thermodynamic data of $1 \cdot \mathrm{G} 51: 3 \mathrm{v} / \mathrm{v} \mathrm{H}_{2} \mathrm{O} / \mathrm{EtOH}$ :
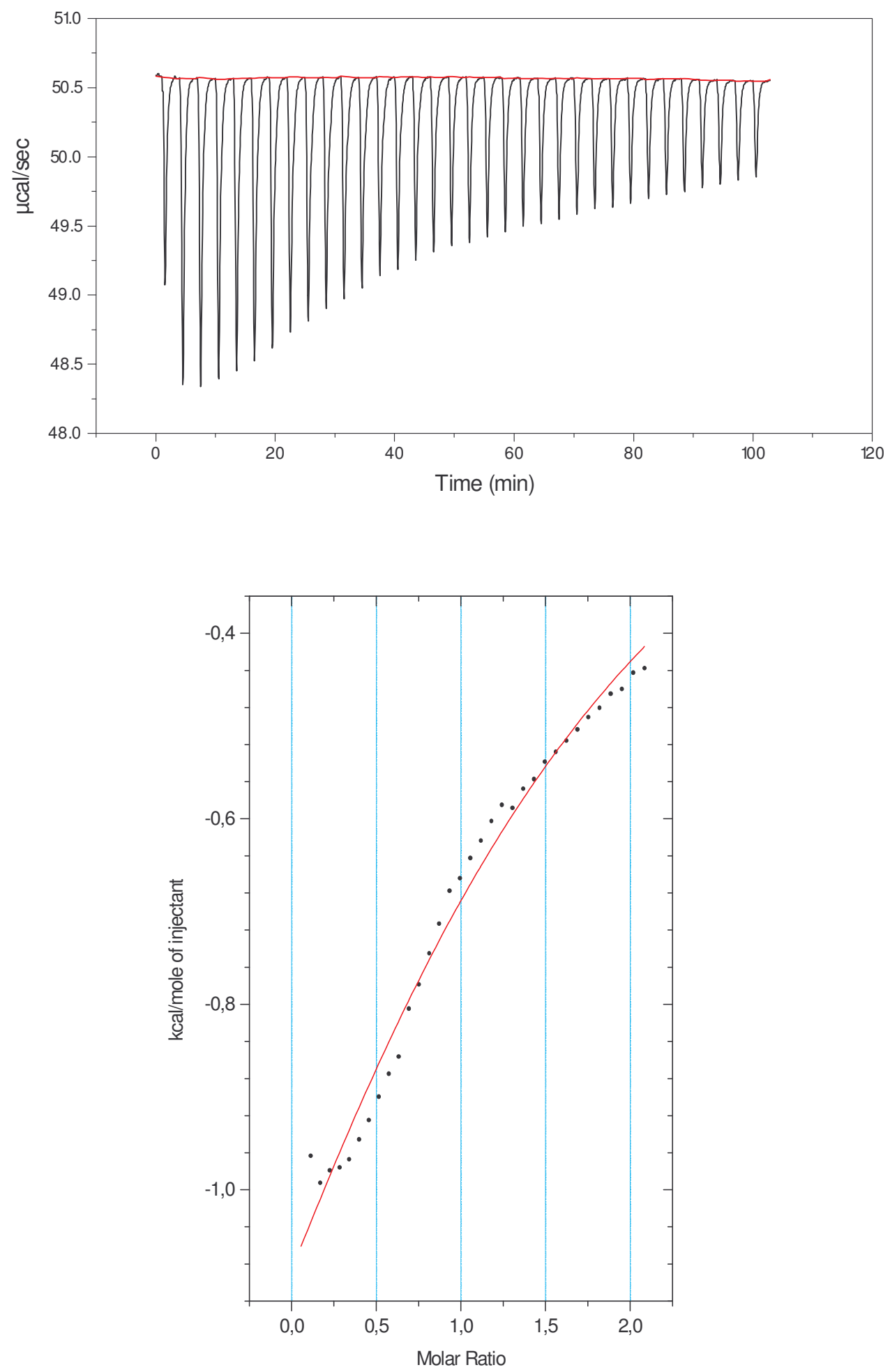

Figure S5 
ITC curve and thermodynamic data of $1 \cdot$ isocitrate $1: 3 \mathrm{v} / \mathrm{v} \quad \mathrm{H}_{2} \mathrm{O} / \mathrm{EtOH}$ (sodium salt):
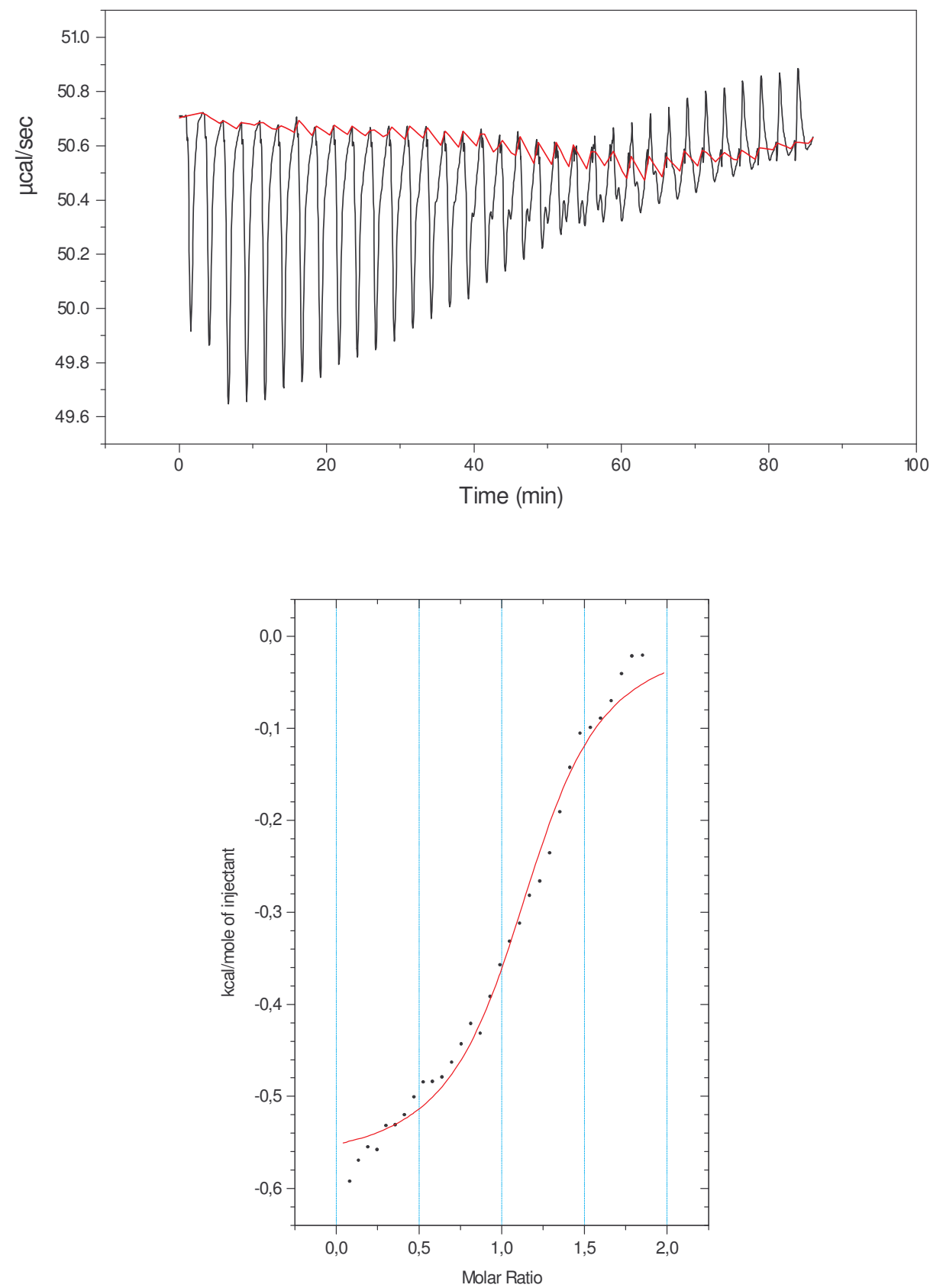

Figure S6 


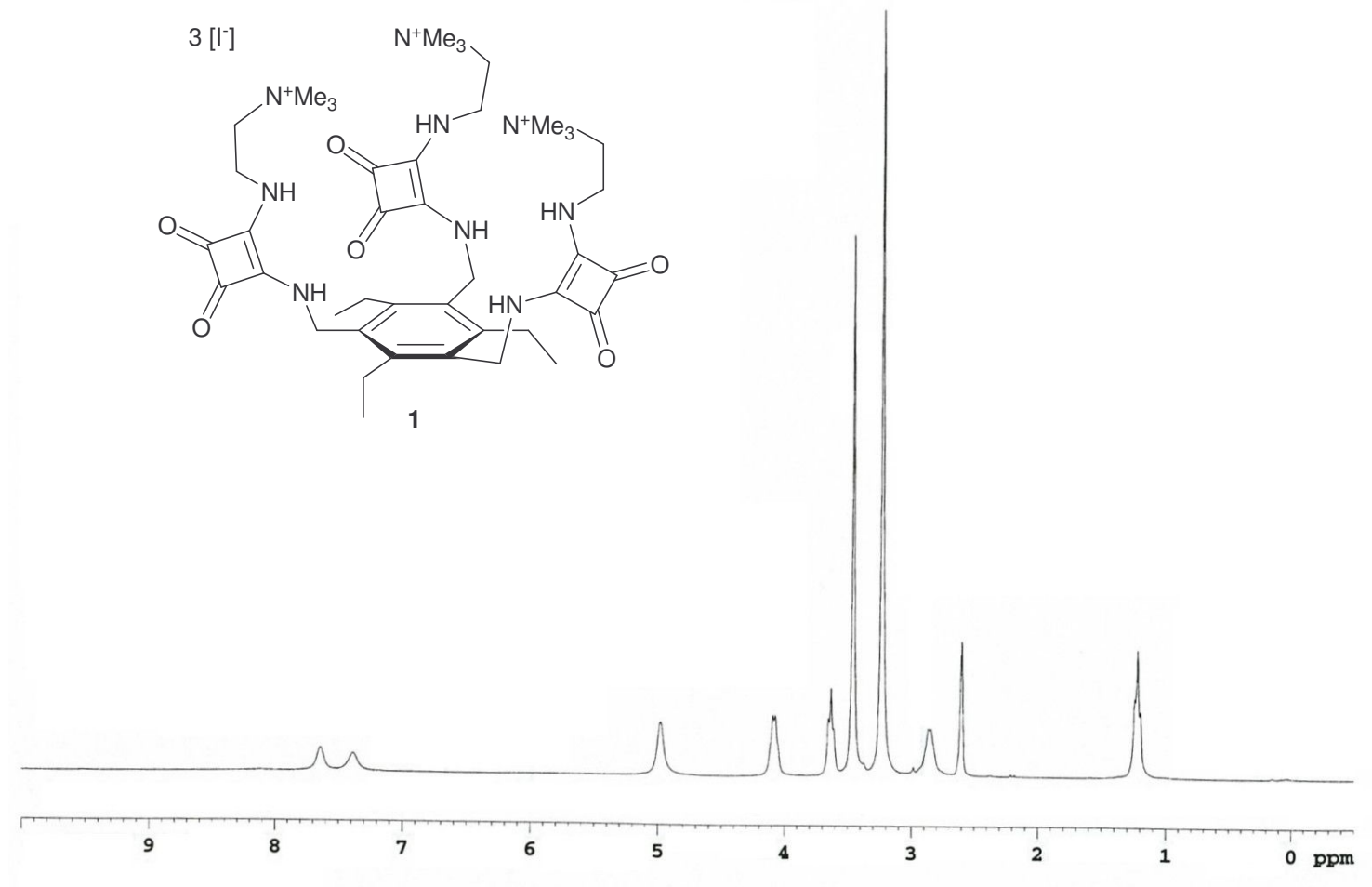

Figure S7. ${ }^{1} \mathrm{H}-\mathrm{NMR}$ of compound 1. 

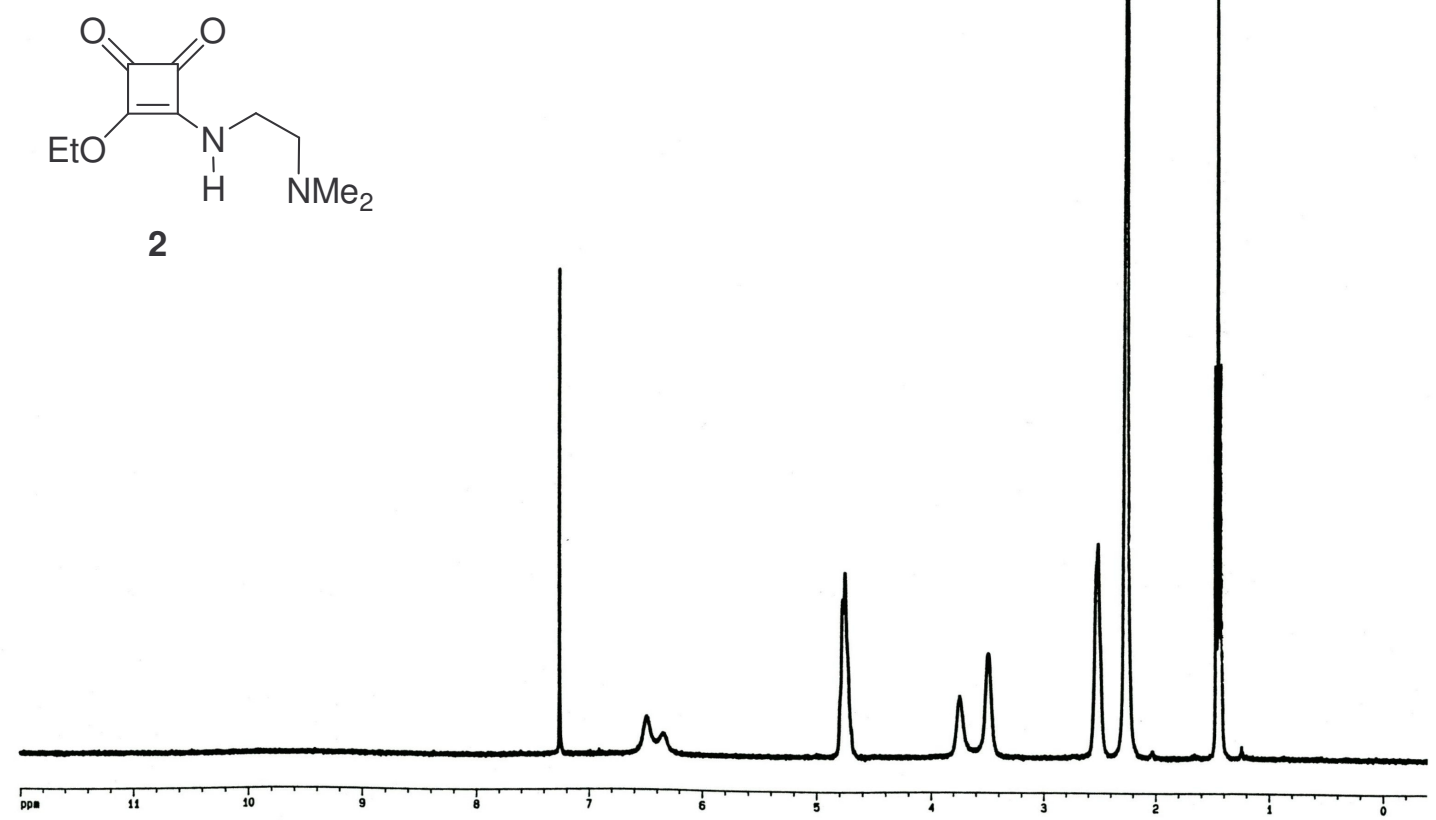

Figure S8. ${ }^{1} \mathrm{H}-\mathrm{NMR}$ of compound 2. 


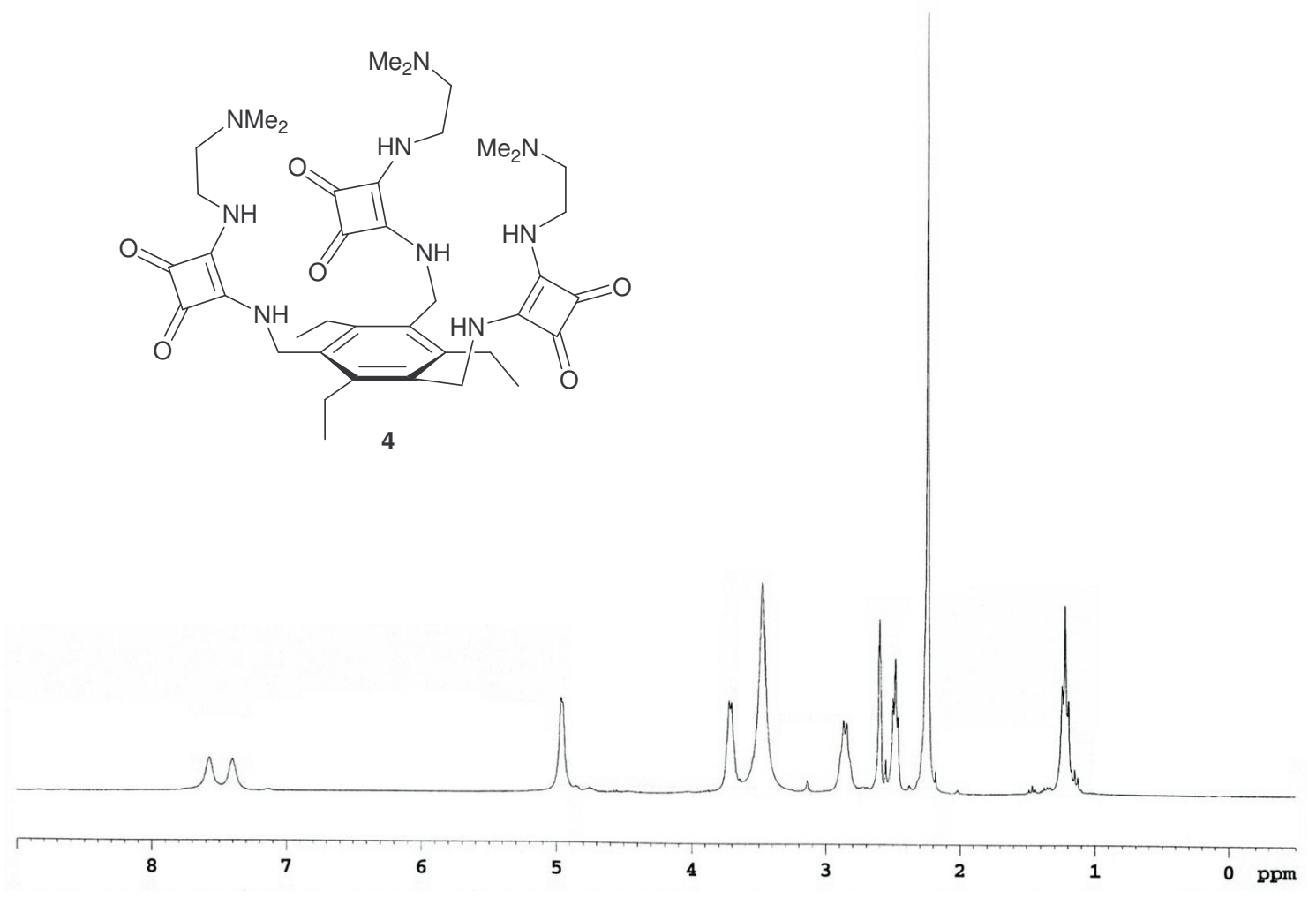

Figure S9. ${ }^{1} \mathrm{H}-\mathrm{NMR}$ of compound 4. 